$1-1-2021$

\title{
The power and process of shifting gender norms: Insights from a randomized controlled trial in South Africa
}

\author{
Nicole Haberland \\ Population Council \\ Julie Pulerwitz \\ Population Council \\ Ann Gottert \\ Population Council \\ Audrey Pettifor \\ Sheri A. Lippman
}

See next page for additional authors

Follow this and additional works at: https://knowledgecommons.popcouncil.org/departments_sbsr-hiv

Part of the Public Health Commons

How does access to this work benefit you? Let us know!

\section{Recommended Citation}

Haberland, Nicole, Julie Pulerwitz, Ann Gottert, Audrey Pettifor, Sheri A. Lippman, Dean Peacock, Lumbwe Chola, Ryan Wagner, Anna M. Leddy, Rebecca West, Mi-Suk Kang Dufour, Jennifer Hove, Rhian Twine, Rhandzekile Mathebula, Dumisani Rebombo, Angelica Pino, F. Xavier Gómez-Olivé, Chodziwadziwa Whiteson Kabudula, Nkosinathi Masilela, Aimée Julien, Kathryn Spielman, and Kathleen Kahn. 2021. "The power and process of shifting gender norms: Insights from a randomized controlled trial in South Africa," Project SOAR Final Report. Washington, DC: USAID I Project SOAR. 


\section{Authors}

Nicole Haberland, Julie Pulerwitz, Ann Gottert, Audrey Pettifor, Sheri A. Lippman, Dean Peacock, Lumbwe Chola, Ryan Wagner, Anna M. Leddy, Rebecca West, Mi-Suk Kang Dufour, Jennifer Hove, Rhian Twine, Rhandzekile Mathebula, Dumisani Rebombo, Angelica Pino, F. Xavier Gómez-Olivé, Chodziwadziwa Whiteson Kabudula, Nkosinathi Masilela, Aimée Julien, Kathryn Spielman, and Kathleen Kahn 


\section{The Power and Process of Shifting Gender Norms: Insights from a randomized controlled trial in South Africa}

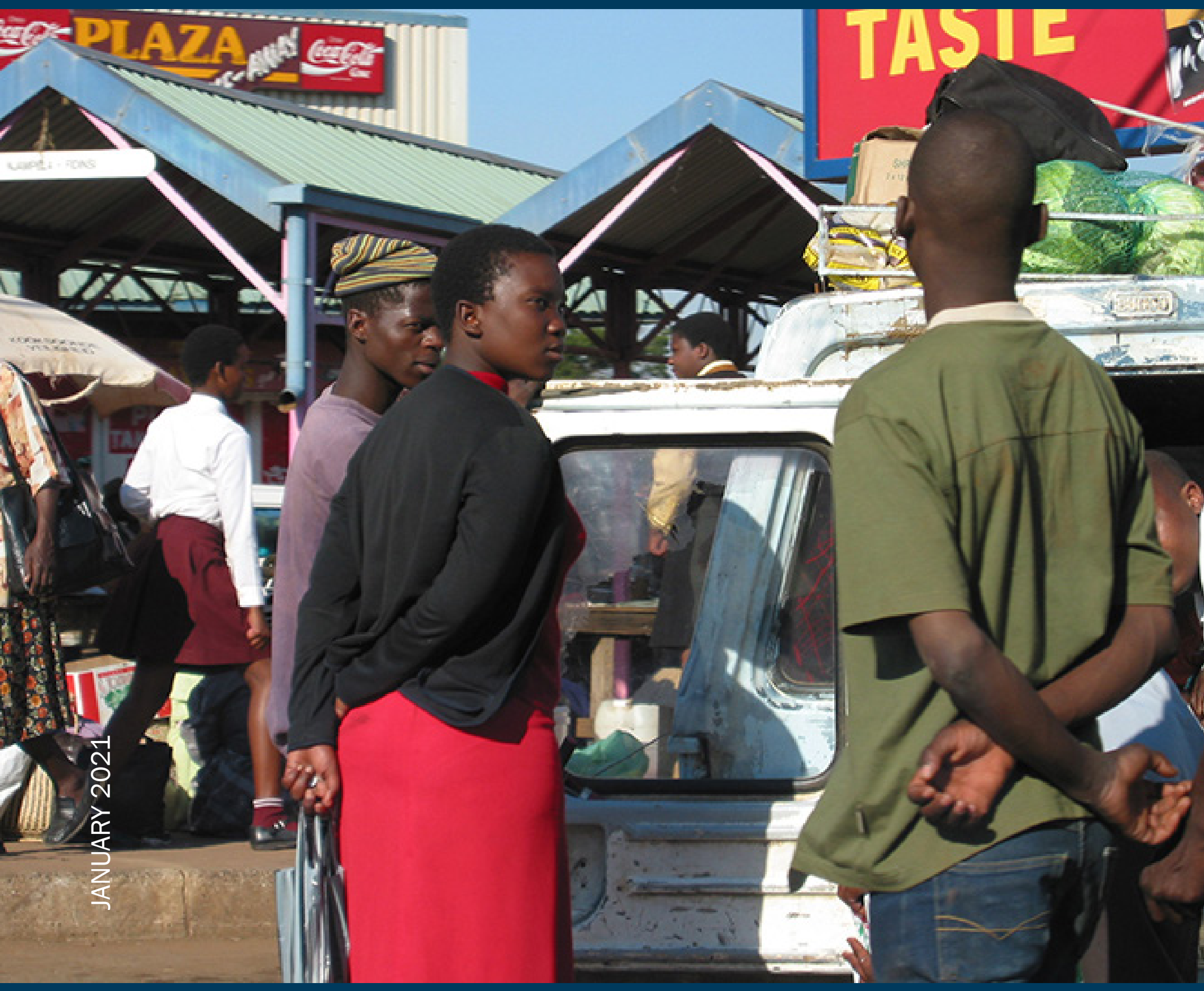

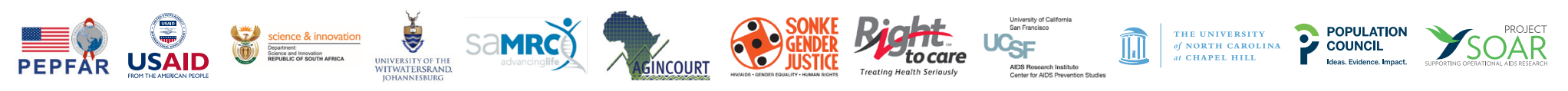


Project SOAR

Population Council

4301 Connecticut Ave, NW, Suite 280

Washington, D.C. 20008 USA

Tel: +1202 2379400

Fax: +1 2022378410

projsoar.org
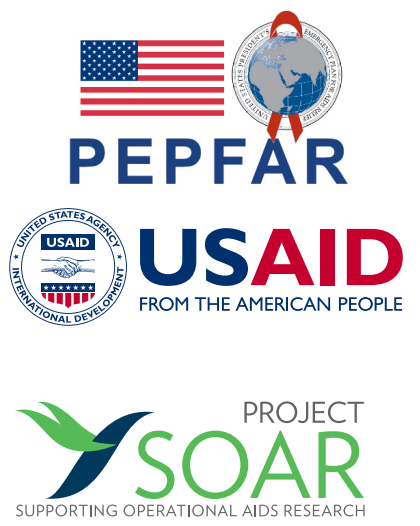

Project SOAR (Cooperative Agreement AID-OAA-A-14-00060) is made possible by the generous support of the American people through the United States President's Emergency Plan for AIDS Relief and the United States Agency for International Development (USAID). The contents of this report are the sole responsibility of Project SOAR and the Population Council and do not necessarily reflect the views of PEPFAR, USAID, or the United States Government.

Through operations research, Project SOAR will determine how best to address challenges and gaps that remain in the delivery of HIV and AIDS care and support, treatment, and prevention services. Project SOAR will produce a large, multifaceted body of high-quality evidence to guide the planning and implementation of HIV and AIDS programs and policies. Led by the Population Council, Project SOAR is implemented in collaboration with Avenir Health, Elizabeth Glaser Pediatric AIDS Foundation, Johns Hopkins University, Palladium, and The University of North Carolina.

The Population Council confronts critical health and development issues-from stopping the spread of HIV to improving reproductive health and ensuring that young people lead full and productive lives. Through biomedical, social science and public health research in about 50 countries, the Council works with our partners to deliver solutions that lead to more effective policies, programs, and technologies to improve lives worldwide. Established in 1952 and headquartered in New York, the Council is a nongovernmental, nonprofit organization with an international board of trustees.

The MRC/Wits Rural Public Health and Health Transitions Research Unit and Agincourt Health and Socio-Demographic Surveillance System, a node of the South African Population Research Infrastructure Network (SAPRIN), is supported by the Department of Science and Innovation, the University of the Witwatersrand, and the Medical Research Council, South Africa.

Cover photo credit: (CPopulation Council

Published in January 2021. (C2021 The Population Council Inc.

Suggested citation: Haberland, Nicole, Julie Pulerwitz, Ann Gottert, Audrey Pettifor, Sheri Lippman, Dean Peacock, Lumbwe Chola, Ryan Wagner, Anna Leddy, Rebecca West, Mi-Suk Kang-Dufour, Jennifer Hove, Rhian Twine, Rhandzekile Mathebula, Dumisani Rebombo, Angelica Pino, Xavier Gómez-Olivé, Chodziwadziwa Kabudula, Nkosinathi Masilela, Aimée Julien, Kathryn Spielman, and Kathleen Kahn. 2021. "The power and process of shifting gender norms: Insights from a randomized controlled trial in South Africa," Project SOAR Final Report. Washington, DC: USAID | Project SOAR. 


\title{
The Power and Process of Shifting Gender Norms: Insights from a randomized controlled trial in South Africa
}

\author{
Nicole Haberland, ${ }^{1}$ Julie Pulerwitz, ${ }^{1}$ Ann Gottert, ${ }^{1}$ \\ Audrey Pettifor,, ${ }^{2,3}$ Sheri Lippman, ${ }^{3,4}$ Dean Peacock, ,6,6 \\ Lumbwe Chola, ${ }^{3}$ Ryan Wagner, ${ }^{3}$ Anna Leddy, ${ }^{4}$ \\ Rebecca West, ${ }^{4}$ Mi-Suk Kang-Dufour, ${ }^{4}$ Jennifer Hove, ${ }^{3}$ \\ Rhian Twine, ${ }^{3}$ Rhandzekile Mathebula, ${ }^{5}$ Dumisani \\ Rebombo, ${ }^{5,8}$ Angelica Pino, ${ }^{5}$ Xavier Gómez-Olivé, ${ }^{3}$ \\ Chodziwadziwa Kabudula, ${ }^{3}$ Nkosinathi Masilela, ${ }^{3}$ \\ Aimée Julien, ${ }^{2}$ Kathryn Spielman, ${ }^{1}$ Kathleen Kahn ${ }^{3}$
}

${ }^{1}$ Population Council

${ }^{2}$ University of North Carolina at Chapel Hill, Department of Epidemiology, Chapel Hill, NC, USA ${ }^{3} \mathrm{MRC} /$ Wits Rural Public Health and Health Transitions Research Unit (Agincourt), University of the Witwatersrand, Johannesburg, South Africa

${ }^{4}$ Division of Prevention Science, Department of Medicine, University of California San Francisco, USA

${ }^{5}$ Sonke Gender Justice, South Africa

${ }^{6}$ Promundo, Washington, D.C., USA

${ }^{7}$ University of Cape Town School of Public Health

8Independent consultant, Johannesburg, South Africa 


\section{ACKNOWLEDGMENTS}

This project would not have been possible without the dedication and outstanding work of the Tsima Community Action Teams and Community Mobilizers. We thank the data collection teams for their hard work. We also thank the Mpumalanga Department of Health for its support and collaboration. We are grateful to Virginia Francis and Nonhlanhla Moyo at the U. S. Agency for International Development (USAID)/ South Africa Mission, and to Amelia Peltz, Sarah Sandison, and Aisha Yansaneh at USAID/DC for their intellectual collaboration and support throughout the project. Our deep thanks to the people living in the Wits/MRC Agincourt study area.

Finally, the Population Council team would also like to take this opportunity to thank our co-authors and staff at Agincourt/Wits, UCSF, and UNC for being such great collaborators and partners. 


\section{TABLE OF CONTENTS}

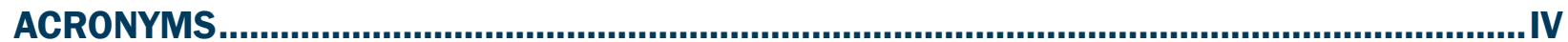

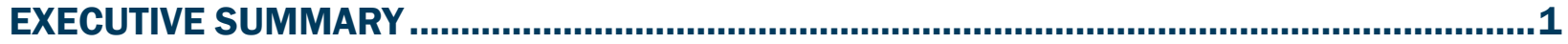

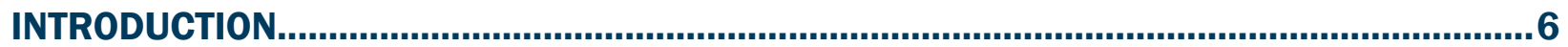

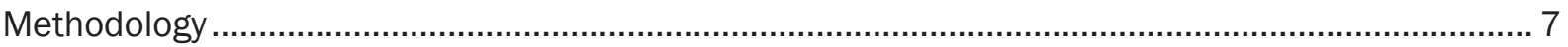

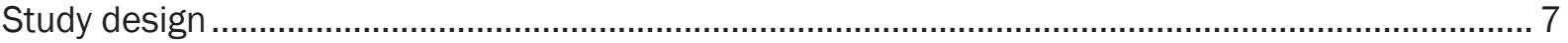

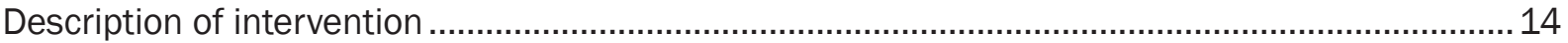

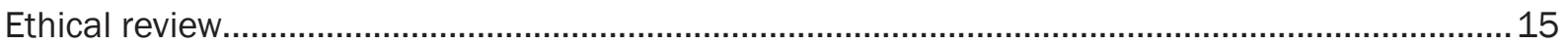

KEY FINDINGS .................................................................................................................. 16

Characteristics of participants and intervention exposure ....................................................... 16

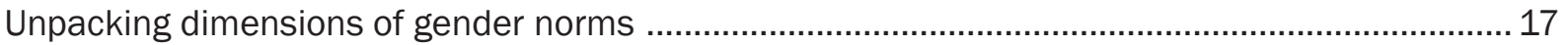

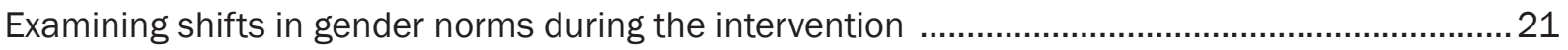

Unpacking gender norm pathways: Intimate partner violence ...................................................29

Unpacking gender norm pathways: HIV testing and treatment ..................................................32

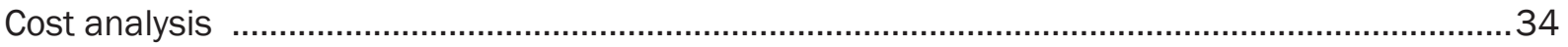

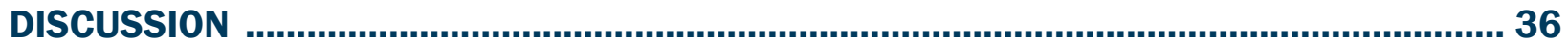

CONCLUSION AND RECOMMENDATIONS................................................................................ 40

REFERENCES ....................................................................................................................... 42 


\section{ACRONYMS}

$\begin{array}{ll}\text { ART } & \text { Antiretroviral treatment } \\ \text { CAT } & \text { Community action team } \\ \text { CM } & \text { Community mobilizer } \\ \text { CM for TasP } & \text { Community mobilization for treatment as prevention } \\ \text { FGD } & \text { Focus group discussion } \\ \text { HDSS } & \text { Health and Socio-demographic Surveillance System } \\ \text { IDI } & \text { In-depth interview } \\ \text { IPV } & \text { Intimate partner violence } \\ \text { ITT } & \text { Intent to treat } \\ \text { RCT } & \text { Randomized controlled trial }\end{array}$

iv - The power and process of shifting gender norms: Insights from a RCT in South Africa 


\section{EXECUTIVE SUMMARY}

While the powerful role that inequitable gender norms play in HIV risk, relationships, and gender-based violence is well established, the effects of gender norms on other aspects of the HIV care continuum are less well understood. These include whether and how endorsement of inequitable and restrictive gender norms affects HIV testing and treatment, which specific dimensions of gender norms are salient for HIV service use and how this differs for women and men, and whether efforts to change gender norms at the community level can lead to increases in HIV testing and treatment and decreases in gender-based violence. We report on findings from a randomized controlled trial of Tsima ra rihanyu ("working together for health"), a threeyear community mobilization program for treatment as prevention (CM for TasP) that aimed to address the social barriers to HIV testing and treatment in Mpumalanga Province, South Africa. In this report we explore the types of gender norms that are salient for women and men, how these relate to HIV testing and treatment, and the pathways through which gender norms operate to affect women's and men's HIV service use and experience and perpetration of intimate partner violence (IPV).

\section{METHODOLOGY}

The study used a cluster randomized controlled design with eight intervention and seven control communities. Findings presented in this report come from three sources: pre-post crosssectional surveys conducted before the three-year intervention began and just after it ended, in all intervention and control communities; qualitative data gathered at three time points over the course of the study (in the beginning, toward the end, and after preliminary analysis of endline results); and a costing analysis to document the costs of the intervention.

Survey participants were men and women ages 18-49 randomly selected from intervention and control villages (final evaluation samples were 1,149 baseline [2014] and 1,189 endline [2019]). Endorsement of equitable/inequitable gender norms was measured using an adapted version of the GEM Scale (Ordinal theta 0.88), including four subdimensions (all ordinal thetas $\geq 0.70$ ) hypothesized to be particularly relevant for HIV testing and treatment:

1. Norms condoning men's violence and control over women

2. Norms around men as decision-maker in the couple

3. Norms around men's toughness and avoidance of help seeking

4. Norms around women's primary responsibility as family caretaker

At baseline, we conducted logistic regression analyses to examine associations between endorsement of equitable gender norms (overall and each sub-dimension) and HIV testing and treatment, controlling for age, marital status, and education. For the evaluation we conducted logistic regression analyses using both baseline and endline survey data, taking an intent to treat (ITT) approach to assess changes in gender norms and IPV over time, as well as differences 
in change over time by study arm (i.e., intervention effect), controlling for age, marital status, education, and employment status.

The qualitative component involved in-depth interviews (IDIs) and focus group discussions (FGDs) conducted with community members, community leaders, and Tsima community mobilizers and community action team (CAT) members (round $1 n=30$; round $2 n=55$; round $3 n=26$ plus two FGDs with 11 community mobilizers). Round 1, conducted in the middle of the three-year intervention, explored prevailing gender norms and gender-related barriers to HIV services. Round 2 was conducted during the last year of the intervention and explored changes in gender norms, IPV, and HIV service use among men and women during the intervention. Round 3 was conducted after we had generated preliminary findings from the evaluation and explored possible explanations for these findings.

The costing was conducted using a retrospective expenditure analysis of project books and accounts for the full project period. Research and intervention costs were separated and only intervention costs were included in the analysis. Costs were estimated for the full intervention, different activity types, and person contacts.

\section{KEY FINDINGS}

\section{The four subdimensions of inequitable gender norms we explored differed in terms of how strongly and widely they were endorsed}

For the baseline survey, both women's and men's responses to a composite GEM Scale and four subdimensions generally reflected endorsement of inequitable gender norms (with lower scores representing less equitable views). Responses to the subdimension regarding women's primary responsibility as family caretaker reflected the most inequitable views (mean of 1.80 on a range of 1.0 to 3.0, for both men and women). The subdimensions relating to men's violence/control and men as decision-makers in a couple also reflected endorsement of inequitable norms. In contrast, responses to the subdimension around men's toughness and avoidance of healthseeking were the most equitable (mean score of 2.51 for women and 2.47 for men).

\section{Endorsement of equitable gender norms was associated with HIV testing and treatment in both anticipated and unanticipated ways}

Analysis of baseline data showed that for women living with HIV, more equitable views on gender norms was significantly associated with increased odds of reported current ART use for the composite GEM Scale (aOR 6.67; $p<0.01$ ), as were three out of four gender norm subdimensions: men's violence and control (aOR 2.94; $p<0.05)$, men as decision-makers in the couple (aOR 5.56; $p<0.01$ ), and men's toughness and avoidance of health seeking (aOR 2.86; $p<0.05)$. Among men living with HIV, equitable scores regarding the subdimension of men as decision-makers in the couple were significantly associated with increased odds of treatment use (aOR3.57; $p<0.05)$. 
Among women, more equitable views on gender norms decreased the odds of reported HIV testing-this finding was significant for the composite score (aOR 0.40; $\mathrm{p} \leq 0.01$ ), as well as norms condoning men's violence ( $\mathrm{aOR} 0.57 ; \mathrm{p} \leq 0.05$ ) and women's primary responsibility as family caretaker (aOR 0.46; $p \leq 0.001$ ). For men there was only one significant association between more equitable views on gender norms and reported HIV testing: men with more equitable scores regarding condoning men's violence and control over women were less likely to report an HIV test in the past year (aOR 0.65; $p<0.05)$.

\section{There were broad, substantial shifts toward more equitable gender norms across control and intervention communities over the study period}

In both intervention and control communities there were significant, large-magnitude (approximately 15\%) increases in GEM Scale scores among both men and women, representing greater endorsement of equitable norms over time. Women's and men's composite GEM Scale score increased (i.e., became more equitable) from baseline to endline in multivariate analysis (adjusted beta for women 0.25; $p<0.001$; adjusted beta for men 0.33; $p \leq 0.001$ ). Women's and men's scores on each of the four subdimensions also significantly increased by roughly similar margins over the course of the study. However, there were no significant differences between study arms, and the change in participant's gender views cannot be attributed to the intervention. Qualitative findings confirmed a shift in gender norms. The round 3 interviews and FGDs, which were conducted after the endline, suggested that the broad shifts may be due in part to rapidly increasing access to TV (via satellite dish) and smartphones across the study area (as elsewhere in South Africa), exposing more people to serial dramas and media that negatively portray IPV and model couples communicating equitably and resolving conflicts.

\section{There are diverse pathways through which changes in gender norms affected IPV and HIV testing and treatment}

The evaluation showed that the intervention led to a significant decrease in women's experience of IPV (aOR 0.53; $p \leq 0.05$ ). Men's reports of IPV perpetration also decreased, but did so in both intervention and control communities so that the change among men was not attributable to the intervention. There were also quantitative findings suggestive of some increases in HIV testing and treatment (Lippman et al., forthcoming). Qualitative data help clarify the pathways through which shifts in gender norms contributed to changes in IPV, as well as HIV testing and treatment uptake. The following themes emerged: 1) There were important changes in gender norms, IPV, and HIV testing, treatment, and disclosure over the study period; 2) Tsima encouraged and built skills around more equitable, constructive communication between intimate partners; 3 ) together, these shifts helped men find alternatives to violence and facilitated treatment uptake and retention; and 4) new knowledge about the benefits of treatment as prevention was more actionable, for example, because of reduced endorsement of inequitable norms regarding men's health seeking and men's toughness. 


\section{Older men were harder to reach with programming and harder to change outcomes}

Attitudes and behaviors appeared to be more difficult to influence among older men (i.e., ages 30-49). Older men were less likely than younger men to participate in Tsima, experienced a smaller magnitude increase in their GEM Scale score in intervention vs. control communities (although at endline, scores were higher, i.e., more equitable, in both), and did not differ over time or between intervention and control communities in terms of reductions in IPV perpetration.

\section{Cost of the intervention}

The estimated total programmatic cost of Tsima was US $\$ 429,000$ for the three-year period 2015 to 2018 -or US $\$ 143,000$ per year-and the average cost was US $\$ 4.68$ per contact. While we are not aware of other cost analyses of community mobilization for HIV prevention and treatment among general populations, the overall annual cost of the project is similar to a community mobilization project to prevent IPV in Uganda (US\$139,000 per year) (Michaels-lgbokwe et al 2017). This study is one of the few cost analyses of community mobilization for HIV prevention in low- and middle-income countries.

\section{CONCLUSION AND RECOMMENDATIONS}

This randomized controlled trial (RCT) demonstrated that shifting norms toward being more gender-equitable can create the enabling environment for significant decreases in IPV and increases in HIV treatment, although it simultaneously raised questions about the ways in which men's low use of HIV services is explained by gender norms. The study demonstrated that shifts in views toward gender can be fostered in a meaningful way at the community level, and that such shifts may also provide an important boost at the individual level. Further, decreases in IPV could be a path to testing for women and men. To apply these results most effectively to programs, we recommend:

1. When implementing gender transformative work to address HIV and IPV, conduct rigorous research to determine the extent to which different gender norms influence what-and whosebehavior and tailor interventions accordingly.

2. Combine activities to shift gender norms with skill building to ensure translation into practices-such as more equitable couple communication and conflict negotiation as a way to prevent IPV and encourage HIV testing and treatment.

3. Simultaneously address gender-related barriers to HIV service use-such as gender norms and IPV that prevent disclosure and treatment seeking-while providing concrete information about the benefits of early testing and treatment and TasP, to ensure informed decision-making about health and to maximize impact on HIV testing and treatment.

4. When certain gender norms are shown not to be a predominant barrier to the use of HIV services for a subpopulation, explore and address other possible impediments to service utilization, such as clinic hours, wait time, concerns about confidentiality, etc., some of which can be gendered issues, but not always. 
5. Conduct additional rigorous evaluation studies designed to explicitly explore implementation questions, including optimal exposure and length of interventions to change gender norms, efficient combinations of intervention components, etc.

6. Follow up participants over the longer term to observe possible additional/ripple effects of interventions like Tsima on, for example, new HIV infections and retention in care.

7. Pay greater attention in strategic planning and intervention development to critical, broader, social changes underway in a program's setting-including rapidly expanding media and internet access across sub-Saharan Africa-and how these changes can be leveraged to improve health behaviors. 


\section{INTRODUCTION}

Endorsement of inequitable gender norms has been linked to multiple adverse health and development outcomes including low rates of condom use, multiple and concurrent sexual partners, intimate partner violence (IPV), STI/HIV incidence, and substance abuse (Tsai and Subramanian 2012; Pulerwitz et al. 2010; Jewkes et al. 2010; Gottert et al. 2017). Consequently, the importance of addressing harmful gender norms is increasingly a part of policy and program discussions in the global HIV field. Yet substantial gaps exist in the literature. The ways in which inequitable gender norms affect HIV testing, linkage to treatment, and retention in care, for example, is underexamined. A recent scoping review of studies on masculine norms and HIV testing found only qualitative studies (Sileo et al. 2018). Despite the absence of quantitative research on the relationship between men's low use of HIV services and norms of manhood, public health programs have been developed to shift men's gender norms to increase their use of HIV testing and treatment. More research exists on the link between IPV and HIV service utilization, with a systematic review and meta-analysis finding IPV to be significantly associated with lower ART use (Hatcher et al. 2015). However, in that review all identified studies were crosssectional, leaving open the question of causality, and whether other unobserved factors were driving the association.

With 90-90-90 goals central to HIV prevention efforts, including early and universal HIV treatment, there is an urgent need to better understand the roles gender norms play in HIV testing and treatment uptake (The INSIGHT START Study Group 2015; Montaner et al. 2014; Cohen et al. 2016; Cohen, McCauley, and Gamble. 2012; Grinsztejn et al. 2014). This includes unpacking specific dimensions of gender norms, and whether and how these differ for women and menfor example qualitative literature suggests that certain masculine norms prevent, and others facilitate, HIV testing (Sileo et al. 2018). Additionally, while programs aiming to address gender norms have demonstrated notable impact on program participants' views on gender (Dworkin, Treves-Kagan, and Lippman 2013; Pulerwitz et al. 2012; Haberland 2015), there is limited evidence of the effectiveness of programs that target the broader community and whether those programs can improve HIV and violence outcomes by shifting gender norms.

South Africa has an estimated 7.5 million people living with HIV (UNAIDS 2019). In 2015 the South African government adopted the 90-90-90 targets as part of its efforts to end the AIDS epidemic (PEPFAR, USAID, and Government of South Africa 2015) and now has the largest HIV treatment program in the world, with over two-thirds of adults living with HIV on ART (UNAIDS 2019). IPV is also endemic, with one in four (26\%) women age 18 or older having experienced physical, sexual, or emotional violence at the hands of a partner, and half (51\%) reporting that their partner displays at least one controlling behavior (National Department 2016).

This study reports on findings from a randomized controlled trial of Tsima ra rihanyu ("working together for health"), a community mobilization program for treatment as prevention (CM for TasP) that aimed to address the social barriers to HIV testing and treatment in Mpumalanga Province in South Africa (Lippman et al. 2017a). While community mobilization as a component of combined 
intervention strategies has been associated with improvements in condom use behaviors and HIV testing uptake (Sweat et al. 2011; Lippman et al. 2017b), the CM for TasP study seeks to address the dearth of rigorously evaluated community mobilization interventions directed specifically at social barriers to HIV-related prevention and care behaviors across the continuum of care. In this report we explore the types of gender norms that are salient for women and men, how these relate to HIV testing and treatment, and the pathways through which gender norms operate to affect women's and men's HIV service use and experience and perpetration of IPV.

\section{METHODOLOGY}

\section{Objectives}

In the context of a randomized controlled trial (RCT) assessing the impact of a community mobilization intervention on HIV testing and retention in care, the SOAR Gender Norms study aimed to explore in-depth the process of gender norms change and the pathways through which such changes affect HIV testing and treatment as well as IPV. The SOAR Gender Norms study assessed the following four objectives:

- Effects of endorsement of equitable gender norms on HIV testing, and the process through which changes in gender norms may affect testing.

- Effects (among those HIV-positive) of endorsement of equitable gender norms on HIV treatment outcomes, and the process through which changes in gender norms may affect treatment.

- Effects of endorsement of equitable gender norms on IPV, and specifically the process through which changes in gender norms may affect IPV.

- Cost of the intervention.

\section{STUDY DESIGN}

\section{Study setting}

This study was conducted in the Agincourt Health and Socio-demographic Surveillance System area (Agincourt HDSS) run by the Medical Research Council/Wits University Rural Public Health and Health Transitions Research Unit, where an annual census has been conducted since 1992 (Kahn et al. 2012). The Agincourt HDSS, with a population of approximately 115,000 , is located in Bushbuckridge sub-district in Mpumalanga Province of South Africa, a largely rural province with among the highest adult HIV prevalence of the country's nine provinces, at 22.8 percent (Human Science Research Council 2018).

\section{Parent study trial and intervention}

Tsima was evaluated via a community randomized controlled trial to test the effects of "Community Mobilization for Treatment as Prevention" (CM for TasP), a three-year mobilizing intervention to increase HIV testing and linkage to and retention in care and treatment among 
men and women ages 18-49 years, by addressing key social barriers to service uptake-including inequitable gender norms. For the trial, eight communities were randomized to receive the intervention, and seven to control/no intervention.

\section{Survey sample}

Quantitative data come from two cross-sectional surveys: 1) a population-based survey conducted in 2014 that served as both an endline assessment for a previous randomized trial (Pettifor et al. 2018; R01MH087118) and as a pre-intervention baseline for the current CM for TasP trial (Lippman et al. 2017a; R01MH103198); and 2) an endline survey conducted for the CM for TasP trial from July to December 2018. The sampling frame consisted of all HDSS households with at least one resident aged 18-49 enumerated during the Agincourt HDSS from 2013 (for baseline) or 2017 (for endline). Only one person was interviewed per household. To select the sample, each household was designated in the male or female sampling frame based on HDSS data (in order to generate adequate sampling frames for females and males in each community), and individuals of that gender in the household were randomly ranked (1, 2, 3, etc.). Upon entering a home, the individual randomly ranked first was screened for the following more detailed eligibility criteria: currently lived in the home, age 18-49 per confirmed date of birth, and had lived in the study area for most of the past 12 months. If the first individual did not meet these eligibility criteria, the second was screened, and so on.

While only 15 of the 27 villages in the HDSS were included in the randomized trial, the surveys, which also aimed to characterize the populations and their participation in the HIV prevention and care continuum, included all 27 villages. As a result, for the cross-sectional baseline analyses ("unpacking gender norms") we used the full 2014 data set that covered 27 villages and included 2,057 respondents, rather than restricting to the $15 \mathrm{CM}$ for TasP study villages. The same survey was administered to all respondents.

For the evaluation analyses we used an intent-to-treat approach. The baseline included only data from the 2014 survey that were collected in the seven control and eight intervention villages for the CM for TasP trial. This provided a final baseline sample of 1,149 participants. The final sample for the 2018 endline survey was 1,189 participants. More details about the samples are presented in the results section.

\section{Survey procedures}

The survey was administered in the participant's home by a trained interviewer, in the local language of Shangaan or in English, based on the participant's preference. The survey generally lasted one to two hours and was administered using computer assisted personal interviewing, in which the interviewer reads each question to the respondent, then enters the answer into an electronic form on a laptop computer.

\section{Measures}

Endorsement of equitable/inequitable gender norms was measured by the GEM Scale (Pulerwitz et al. 2008), which had been previously validated in the study site (Gottert et al. 2016). To the extent that aggregation of individuals' endorsement of gender equitable norms is indicative of the 
existence of a norm in the community, we used the GEM Scale score and its subdimensions as a proxy for gender norms in the community.

Both original GEM Scale items as well as approximately 15 new items tailored to this study were included in the survey. Response categories for each of the 40 items were "Agree a lot," "Somewhat agree," and "Do not agree at all."

We selected items from among the 40 that, based on the literature, reflect specific dimensions of gender norms hypothesized to be salient for HIV testing and treatment. These included:

- Norms condoning men's violence and control over women (7 items; e.g., "A man is expected to discipline his woman").

- Norms around men as decision-maker in a couple (6 items, e.g., "A man should have the final word about decisions in his home").

- Norms around men's toughness and avoidance of health-seeking (5 items, e.g., "For men, getting sick is a sign of weakness").

- Norms around women's primary responsibility as family caretaker (5 items, e.g., "A woman's role is taking care of her home and family").

To arrive at each final set of items, we removed one to two originally-included items based on low factor loadings (<0.30). We constructed a "composite," adapted, GEM Scale variable comprising all items across the sub-dimensions ( 23 items), as well as variables for each subdimension (see Table $\mathbf{G}$ in the Results section for the final composite scale and subdimension items).

We constructed scale scores by taking the mean of non-missing items and multiplying by the number of items in the set, with scores ranging from 1.0 to 3.0 and higher scores representing more equitable views on gender norms. The composite scale as well as each sub-dimension had good internal reliability, with ordinal theta (similar to Cronbach's alpha) (Zumbo et al. 2007)) of 0.88 for the composite score and $\geq 0.70$ for each sub-dimension (Pulerwitz et al. 2019).

IPV perpetration (among male respondents) or IPV experience (among female respondents) was defined as reporting perpetrating or experiencing at least one of seven types of physical or sexual IPV on a World Health Organization questionnaire adapted for South Africa (Dunkle et al. 2006; Jewkes et al. 2010). Only individuals who had an intimate partner were asked these questions and included in analyses.

HIV testing in the last year was a binary variable defined as having at least one HIV test in the last 12 months (with any HIV-positive respondents who had known their status for longer than a year removed from the denominator). Current ART use, assessed among respondents self-reporting as HIV-positive, was a binary variable constructed based on an affirmative answer to two questions: "Are you on antiretroviral treatment or ART now or were you ever on ART?" and "Are you still taking ART?"

Covariates included age (kept as continuous in regression models), marital/cohabiting status (binary), educational status (binary: completed at least secondary education vs. lower), and employment status (binary: assessed by the question "have you earned any income in the last 3 months?"). 
Survey and intervention variables: Rounds were designated as 0 (baseline) or 1 (endline). Intervention status was designated 0 (resides in control community) or 1 (resides in intervention community).

\section{Statistical analysis}

All data analyses were performed using Stata v15 (StataCorp 2017) and were conducted separately for men and women. All analyses were weighted to account for sampling probability and to represent the distribution of men and women age 18 to 49 in Agincourt based on Agincourt HDSS censuses conducted in 2013 (for 2014 baseline) and 2017 (for 2018-19 endline). In addition, we used robust standard errors to account for clustering by village (StataCorp 2017).

For the baseline analyses (unpacking gender norms) we generated weighted means and proportions for variables of interest, and conducted weighted logistic regression, accounting for the cluster sampling design, to examine associations between the full GEM Scale (composite), and each of the four sub-dimensions, with last-year HIV testing and current ART use.

Evaluation analyses were performed both on the full age range, and for ages 18-29 and 30-49 years separately. For the evaluation models, we included the intervention status (intervention or control), the round (baseline or endline), and the interaction between intervention and round. We then examined the effect estimate for round (re: change over time regardless of intervention status), as well as the interaction term, which reflects the difference in changes over time by study arm (i.e., ITT effect). We adjusted the models for demographic variables hypothesized to be associated with outcomes: age, education, marital status, and employment status, accounting for the cluster sampling design. For this report we do not present the effects of degree of exposure to the intervention (sometimes called "per protocol" analyses); these data are available on Dataverse).

\section{Clinic data}

While the quantitative analyses in this report draw from the baseline and endline surveys that include information on gender norms, SOAR also supported the collection of clinic data for the NIH parent study. All local, public clinics in the study site offer HIV testing and treatment. Wits' Clinic Link system, which is an electronic data capture system set up across all clinics located within the HDSS, allowed for clinical data to be linked with the Agincourt HDSS census. The clinic link data system, therefore, provides a platform to assess service delivery for every resident attending public clinics across the HDSS. The clinic link data will be used to assess whether the Tsima program increased overall HIV testing and care in intervention village residents as compared to control village residents. Using service delivery information provides an objective data source for trial outcomes, while the survey provides more detailed information on social barriers to care experienced. During our program, we were also able to capture mobile clinic and other project-sponsored events where HIV testing took place. Any study village residents who sought services at private clinics or clinics outside of the study site would not be captured in the clinic link data system. 
To support the clinic link activities, trained research assistants and data entry staff consented adult clients seeking HIV services in all public health facilities in the study site to link their clinical data to the census database. Sites included 10 clinics at the beginning of the study and, when two clinics merged in 2017, nine clinics thereafter. Once clients consented to linkage, their chart data were extracted. Supervisors conducted regular Clinic Link data audits to ensure quality data. Clinic data were then linked with HDSS census data. Findings from these analyses will be presented in the final parent study paper which will be available shortly and are not covered in this report. As soon as the paper becomes available, we will include the link here/post the link on the SOAR project website.

\section{Qualitative sample, procedures, and analysis}

There were three rounds of qualitative data collection: March-April 2017 about halfway through the intervention (round 1), March-May 2018 toward the end of the intervention (round 2), and March-April 2019 after completion of the quantitative endline survey and preliminary endline analyses (round 3). In the first two rounds, we conducted in-depth interviews (IDIs) with intervention staff ( $n=23$, community mobilizers and CAT members), community opinion leaders $(n=7)$, and, in round 2 , an additional 25 community members $(n=25)$. For IDIs with community members at round 2 , we recruited roughly equal numbers from different categories made up of combinations of the following: male vs. female, HIV-negative vs. HIV-positive, tested for HIV in last year vs. not (among HIV-negative), and on ART vs. not (among HIV-positive individuals). In addition, to better explicate endline survey findings, the year after the intervention ended we conducted IDIs with community members $(n=26)$ and two FGDs with community mobilizers (total $n=11$ ). For the sample of community members at round 3 , we recruited community leaders (political or traditional) and owners/managers of popular social venues in both intervention and control villages, as well as other community members who had participated in the intervention (specifically, those who reported taking up HIV services vs. not, on the endline survey).

We developed semi-structured IDI and FGD guides for each round, which were used in all interviews/discussions. Guides for the IDIs conducted in round 1 asked about prevailing gender norms and gender-related barriers to HIV services. In round 2, IDI guides asked about changes in gender norms and IPV among men and women over the last three years, including effects of the intervention, as well as changes in HIV service use. Guides for the IDIs and FGDs in round 3 were developed after we had generated preliminary findings from the ITT and exposure analyses using baseline and endline survey data. Those guides explored what we hypothesized-based on reviews of the literature and discussions with local stakeholders as well as the qualitative interviewer team-to be possible explanations for the outcomes. Questions proceeded in such a way as to explore each possible cause in an unbiased/non-leading manner (so as to minimize bias in results) before proceeding to ask more direct questions about it.

Interviews with community mobilizers were held in private at the Tsima or study office or another private location of the participant's choosing; interviews with community members were held in participants' homes or another location of their choosing. IDIs were conducted in English or Shangaan, based on the participant's preference. FGDs were conducted in English by two of the study team, with translation between Shangaan and English by an interpreter, when necessary. All IDIs and FGDs were audio-recorded, transcribed, and translated (as necessary) from Shangaan 
into English. Transcripts were reviewed by the study team for any errors or lack of clarity, and corrected as necessary, in collaboration with the original interviewer. We entered final transcripts into Atlas.ti v7 software for coding and analysis. We then reviewed code reports, with constant comparison between community members, intervention staff, and other key informants (e.g., community leaders), and used thematic analysis as a framework to arrive at final themes (Patton 2015).

\section{Costing}

Costing was undertaken from a provider perspective, using a retrospective expenditure analysis of the project books of accounts, for the full project period May 2015 to July 2018. The cost analysis was guided by the Global Health Costing Consortium (GHCC) reference case, which sets standards for global health costing studies (GHHC 2018). The provider perspective was taken to inform policy on social mobilization for HIV prevention, because the intervention does not lend itself to estimation of family or patient costs. Thus, only costs of implementing the intervention (such as personnel salaries, vehicle operating costs, and equipment) were included, and any costs to individuals attending Tsima activities were not considered.

Full financial and economic costs were estimated, where financial costs were the actual monetary outlays incurred in the purchase of items, and economic costs included the opportunity cost of resource use, for example the valuation of donated goods (UNAIDS 2000). Costs were classified as capital or recurrent costs. Recurrent costs included items such as stationery, fuel, utilities, and personnel time. Capital costs included items such as vehicles, computers, and furniture, and other items whose useful life was more than a year. Capital costs were annuitized to reflect their annual value. The annual financial cost of capital items was calculated using a straightline depreciation method, where the total cost of an item was divided by its useful life years. The annual economic cost of capital items was calculated using a discount rate of 6.75 percent (South African Reserve Bank 2018). Useful life years of seven years were used for furniture, and five to six years for office equipment (National Treasury 2018).

We separated research and intervention costs; only intervention costs are included in this analysis, as costs due to extensive data collection for a randomized trial would not be incurred should the Department of Health choose to replicate or implement the intervention. Since the cost analysis was done retrospectively, time spent on different activities by personnel who worked on both the intervention (program) and evaluation (research) was divided using percent effort, which was determined through interviews with project staff. Other joint costs such as vehicles were allocated between research and intervention costs using percentage usage (also estimated through interviews with staff).

We undertook activity-based costing, where we first identified the main cost centers and then allocated resources (personnel, equipment, furniture, etc.) to each cost center or cost category. The main cost categories were: Start-up, Training, Supervision, and Mobilization Activities.

Start-up costs included all preparatory activity costs such as program development, adaptation of manuals, training, workshops, and initial community engagement activities. Start-up costs were treated as capital costs with a 3-year useful lifespan, equivalent to the duration of the project. Training included all post start-up re-training and other training activities for community 
mobilizers (CMs), who led intervention, implementation, and volunteer community action team members (CATs). Training costs were treated as recurrent costs. The cost of the initial training of CMs and CATs, however, was considered a start-up cost. Supervision included salaries of two supervisors, who oversaw the implementation of the intervention. The supervisor costs were allocated 100 percent to the intervention. Salaries of the driver and study coordinator were included at 50 percent. Other costs included transport, equipment, and costs of all activities related to overall supervision of CMs and CATs during the implementation of the intervention.

Mobilization activities were grouped into three categories-outreach and small group activities, workshops, and events. Outreach and small group activities included door to door outreach, digital stories, ambush theatre, mural painting, support groups, and young women's discussion groups. Workshops included two-day and half-day workshops that included an average of 30 participants per workshop session. Events included larger community-wide events such as street soccer, sports tournaments, and car washes where multiple mobilization activities were conducted within the setting of a larger event. We estimated costs of mobilization activities by type, first by allocating immediate inputs and resources to a specific mobilization activity (outreach, workshops, events); then, for costs that could not be directly attributed to a specific activity, such as salaries, overhead, and transport, we distributed these proportionally based on the number of persons reached by the activity type.

Included in the costs of Mobilization Activities were all items related to community mobilization activities, such as catering for workshops, travel, materials, and supplies used during specific events. Also included were salaries of CMs, since they spent 100 percent of their time supervising CATs and conducting mobilization activities. There were $18 \mathrm{CMs}$ ( $2 \mathrm{CMs}$ per intervention village [n=8] and 2 CMs for Young Women's Groups which ran from March 2017 to July 2018), each fully employed to work on the intervention. CATs were volunteers and not full-time project employees; throughout the project period there were approximately 91 active CAT members (Year 1-22, Year 2-42, Year 3-27).

Project overhead such as such as rent, electricity, telephone, and internet were first allocated equally between evaluation (research) and intervention, and then distributed equally across the cost categories. All costs were adjusted to 2018 prices using a Consumer Price Index (Stats SA 2018). Prices of items were collected in South African rands (ZAR) and then converted to United States dollars (US\$) at the average exchange rate for the year in which costs were incurred (OANDA 2019).

Outcomes: We estimated costs for the following outcomes: full intervention, activity type (outreach, events, workshops), and person contacts. For the latter, we used monitoring data collected by CMs throughout the project. CMs recorded attendance and number of contacts every day they worked and submitted log sheets at weekly meetings, and data typists fed data into monthly and quarterly reports. The outcomes used in this analysis were total number of contacts recorded at events and activities, i.e., the estimated attendance at any Tsima event. While attendance and number of contacts were documented and/or estimated by CMs and CATs, close supervision and guidance make significant misreporting unlikely. 
Calculating total and average costs: Average costs were calculated as total costs divided by total outcomes. We provide cost per contact (total costs/outcomes), as well as the average cost of each activity, i.e., outreach, workshops, and events. The average cost of each activity was expressed as the total activity costs divided by the total attendance for each activity group. To estimate the total activity costs, we summed the start-up, supervision, and training costs and spread these equally across the mobilization activities.

\section{DESCRIPTION OF INTERVENTION}

The intervention, Tsima ra rihanyu ("working together for health"), was based on a defined mobilization model and implemented by Sonke Gender Justice, a South African nongovernmental organization. Described in detail elsewhere (Lippman et al. 2017a), Tsima is based on the theory that the barriers to broad use of treatment as prevention are primarily social and structural, and that to create social change requires community mobilization to address "social barriers to testing, and linkage to and retention in HIV care, specifically, poor awareness or understanding of HIV care; fear and stigma associated with HIV infection, clinic attendance, and disclosure; lack of social support; and gender norms, particularly those that deter men from accessing care" (p 4). The main structure and content of Tsima were designed as part of the parent study, with SOAR contributing to strengthening gender norms-focused messages and activities, in particular, weaving in more attention to females and the gender norms and gendered practices that undermine women's health and wellbeing. This included adding activities that emphasize females' points of view (for example, adding lessons in the two-day workshop agendas that cover intimate partner violence, the continuum of sexual coercion) and building in young women's groups

Figure 1 Tsima intervention manuals

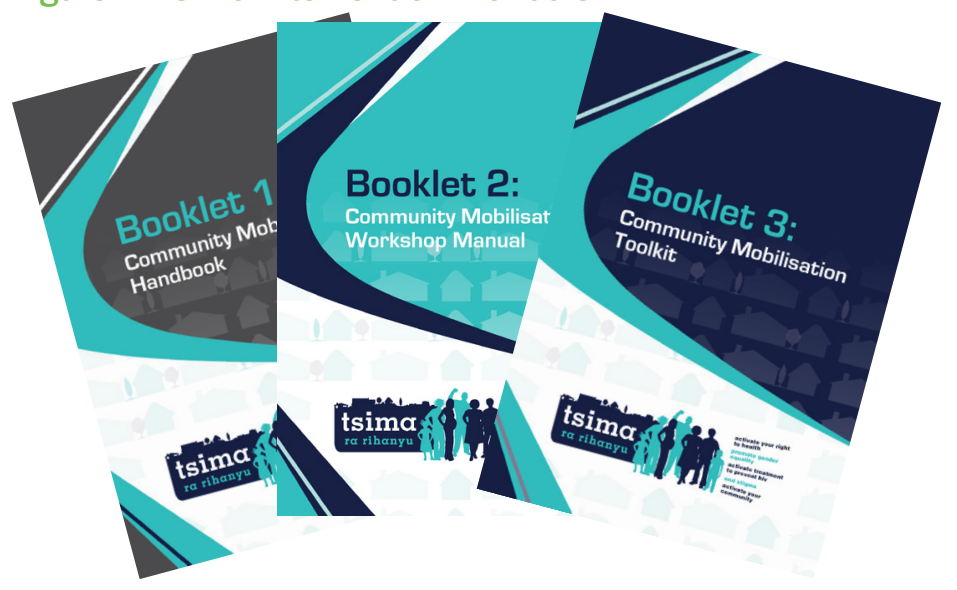
into the array of Tsima activities.

Intervention staff included two program managers, a team of 16 community mobilizers (CMs), and volunteer community action teams (CATs). Intervention content covered six themes:

1. Gender, power, and health;

2. HIV prevention: Community knowledge is community power;

3. Treatment literacy;

4. Healthy communication, healthy relationships;

5. Human rights, HIV, and stigma; and

6. Taking action for change.

Tsima CMs and CATs conducted a range of activities, such as two-day workshops (mixed-gender), 
soccer or other community events, digital story screenings, young women's groups, support groups for people living with HIV, and engaging village leadership and other stakeholders.

Over the course of the three-year intervention, over 9,000 activities and 1,000 CAT meetings were conducted in the eight intervention villages. The vast majority of activities (over 8,000) were outreach/small group activities such as door-to-door education, mural painting, open houses, and health talks. Other activities included 35 young women's groups that met approximately nine times each and reached 421 young women; 591 leadership/stakeholder engagement meetings; 46 events such as soccer tournaments, men's events, and fun days; and 373 two-day workshops.

\section{ETHICAL REVIEW}

The study was approved by the institutional review boards at the University of California-San Francisco, the University of North Carolina at Chapel Hill, the Human Research Ethics Committee at the University of the Witwatersrand in South Africa, and the Mpumalanga Department of Health and Social Development Research Committee. 


\section{KEY FINDINGS}

\section{CHARACTERISTICS OF PARTICIPANTS AND INTERVENTION EXPOSURE}

As noted in the Methods, we conducted analyses with both the full baseline sample of 27 villages and with the baseline and endline data from only the 15 study villages. We describe these samples separately in this section. Table 1 shows the characteristics of the full baseline sample used in baseline analyses. The mean age was 31.5 years for women and 29.4 years for men. About onethird of women and one-quarter of men were
Table 1 Sociodemographic characteristics of participants (extended baseline sample)

\begin{tabular}{|lcc|}
\hline & $\begin{array}{c}\text { Women } \\
(n=1,053) \\
\%\end{array}$ & $\begin{array}{c}\text { Men } \\
(n=1,004) \\
\%\end{array}$ \\
\hline Age (mean) & 31.5 years & 29.4 years \\
Married (vs other) & 39 & 23 \\
Completed high school & 32 & 31 \\
$\begin{array}{l}\text { Received any income } \\
\text { in the past 3 months }\end{array}$ & 36 & 32 \\
\hline
\end{tabular}

married. About one-third of participants, females and males, had completed high school. Thirtysix percent of women and 32 percent of men reported receiving any income in the past three months.

Table 2 presents characteristics of female and male participants in intervention and control villages at baseline and at endline. For the most part, demographic characteristics of samples were equivalent between intervention and control, and in endline compared to baseline surveys. Significant differences included the following: high school completion (for women between baseline and endline, and for men between arms at endline); marital status among men (differences between study arms at endline); and employment among women (between study arms at endline and between baseline and endline). To account for the differences between the samples, we controlled for demographics in our analyses.

There was a robust degree of participation in Tsima activities reported in intervention villages among both men and women and minimal contamination, or reporting of participation in Tsima activities, in control villages. Among respondents in intervention villages, 56 percent of women and 64 percent of men had ever heard of Tsima or seen the logo (data not shown). In control villages, about 15 percent of men and women had heard of Tsima or seen the logo. Among these, contamination was minimal-for example, fewer than five men and five women in control villages reported attending a Tsima workshop. Among individuals in intervention villages who had heard of Tsima/seen the logo, about half of men and women had participated in a workshop in the previous 2 years. Nearly two-thirds of women and men reported that due to Tsima they had talked with other people about testing/treatment, and over half said they had talked with others about issues around gender or relationships between men and women. Over one-third of women and nearly half of men reported feeling part of Tsima. 
Table 2 Participant characteristics by sex, study arm, and survey round

\begin{tabular}{|c|c|c|c|c|c|c|c|}
\hline $\begin{array}{l}\text { Demographic } \\
\text { characteristics }\end{array}$ & $\begin{array}{c}\text { Control } \\
n=539 \\
\text { Weighted } \\
\%(95 \% \mathrm{Cl})\end{array}$ & $\begin{array}{l}\text { Baseline } \\
\text { Intervention } \\
\quad n=610 \\
\text { Weighted \% } \\
(95 \% \mathrm{Cl})\end{array}$ & p-value & $\begin{array}{c}\text { Control } \\
n=558 \\
\text { Weighted \% } \\
(95 \% \mathrm{Cl})\end{array}$ & $\begin{array}{l}\text { Endline } \\
\text { Intervention } \\
\qquad n=631 \\
\text { Weighted \% } \\
(95 \% \mathrm{Cl})\end{array}$ & p-value & $\begin{array}{l}\text { p-value for } \\
\text { diff by round } \\
\text { (irrespective } \\
\text { of } \\
\text { intervention) }\end{array}$ \\
\hline WOMEN & $\mathrm{n}=\mathbf{2 7 4}$ & $n=309$ & & $n=300$ & $n=348$ & & \\
\hline Age (mean) & $\begin{array}{c}33(32.0 \\
33.5)\end{array}$ & $\begin{array}{c}33(31.7 \\
33.6)\end{array}$ & NS & $\begin{array}{c}33(32.8, \\
33.5)\end{array}$ & $\begin{array}{c}33(32.6 \\
33.6)\end{array}$ & NS & NS \\
\hline Completed high school & $\begin{array}{c}30(25.0, \\
36.2)\end{array}$ & $\begin{array}{c}27(19.7 \\
36.0)\end{array}$ & NS & $\begin{array}{l}48(42.2 \\
53.6)\end{array}$ & $\begin{array}{c}44(33.0 \\
55.2)\end{array}$ & NS & $* * *$ \\
\hline Married (vs. other) & $\begin{array}{l}41(35.7 \\
47.0)\end{array}$ & $\begin{array}{c}42(37.2 \\
46.8)\end{array}$ & NS & $\begin{array}{c}39(34.8, \\
43.4)\end{array}$ & $\begin{array}{c}35(25.8, \\
45.0)\end{array}$ & NS & NS \\
\hline $\begin{array}{l}\text { Received any income in } \\
\text { past } 3 \text { months }\end{array}$ & $\begin{array}{c}33(26.7 \\
40.9)\end{array}$ & $\begin{array}{c}40(34.9 \\
45.4)\end{array}$ & NS & $\begin{array}{c}54(51.6 \\
57.1)\end{array}$ & $\begin{array}{c}45(38.4 \\
50.8)\end{array}$ & $* *$ & * \\
\hline MEN & $n=265$ & $n=301$ & & $n=258$ & $n=283$ & & \\
\hline Age (mean) & $\begin{array}{l}30(28.0 \\
31.2)\end{array}$ & $\begin{array}{c}29(27.9 \\
29.4)\end{array}$ & NS & $\begin{array}{c}30(28.6 \\
30.5)\end{array}$ & $\begin{array}{c}29(28.2 \\
30.4)\end{array}$ & NS & NS \\
\hline Completed high school & $\begin{array}{c}31(24.4 \\
37.3)\end{array}$ & $\begin{array}{c}36(26.3 \\
46.8)\end{array}$ & NS & $\begin{array}{l}48(44.5 \\
51.5)\end{array}$ & $\begin{array}{c}35(30.7 \\
40.3)\end{array}$ & $* * *$ & NS \\
\hline Married (vs. other) & $\begin{array}{l}20(15.5 \\
26.0)\end{array}$ & $\begin{array}{c}26(22.4 \\
30.7)\end{array}$ & NS & $\begin{array}{l}17(13.1 \\
21.5)\end{array}$ & $\begin{array}{c}24(19.5 \\
29.2)\end{array}$ & * & NS \\
\hline $\begin{array}{l}\text { Received any income in } \\
\text { past } 3 \text { months }\end{array}$ & $\begin{array}{c}28(23.0 \\
33.5)\end{array}$ & $\begin{array}{c}36(27.1 \\
46.7)\end{array}$ & NS & $\begin{array}{c}31(27.8 \\
33.7)\end{array}$ & $\begin{array}{c}38 \text { (30.1, } \\
45.6)\end{array}$ & NS & NS \\
\hline
\end{tabular}

NS=non-significant; ${ }^{*} p<0.05, * * p<0.01, * * * p<0.001$

\section{UNPACKING DIMENSIONS OF GENDER NORMS ${ }^{1}$}

To better understand the dimensions of gender norms, their mutability, and their salience for improving HIV testing and treatment outcomes, we "unpacked" specific gender norms by topic area and examined how widely they were held by women and men (Pulerwitz et al. 2019). Using data from the full baseline sample, participants' endorsement of equitable gender norms is shown in Table 3 for four gender subdimensions and for a composite GEM Scale score. We found that at baseline, both men and women generally held more inequitable rather than equitable views about gender roles and behavior. The most gender-inequitable domain was related to women's primary responsibility as family caretaker. Within that subdimension, only 25 percent of women and men did not agree with the statement, "A woman's role is taking care of her home and family." The subdimension with the most equitable (highest) scores was men's toughness and avoidance of health-seeking. In this subdimension, participants reported very high disagreement with statements reflecting beliefs that men shouldn't seek health care (e.g., "A man shouldn't go to the doctor unless his situation is serious") but still about half of male and female participants agreed with the norm that men should be tough. The remaining two subdimensionsmen's violence/control and men as decision-makers in a couple-fell in between.

${ }^{1}$ The data and tables in this section and the following are drawn from Pulerwitz et al. (2019); please use the journal article as primary citation source. 
Table 3 Views on gender norms among participants in the baseline sample (27 villages; $\mathrm{n}=1,053$ women; 1,004 men) GEMS composite and subdimensions

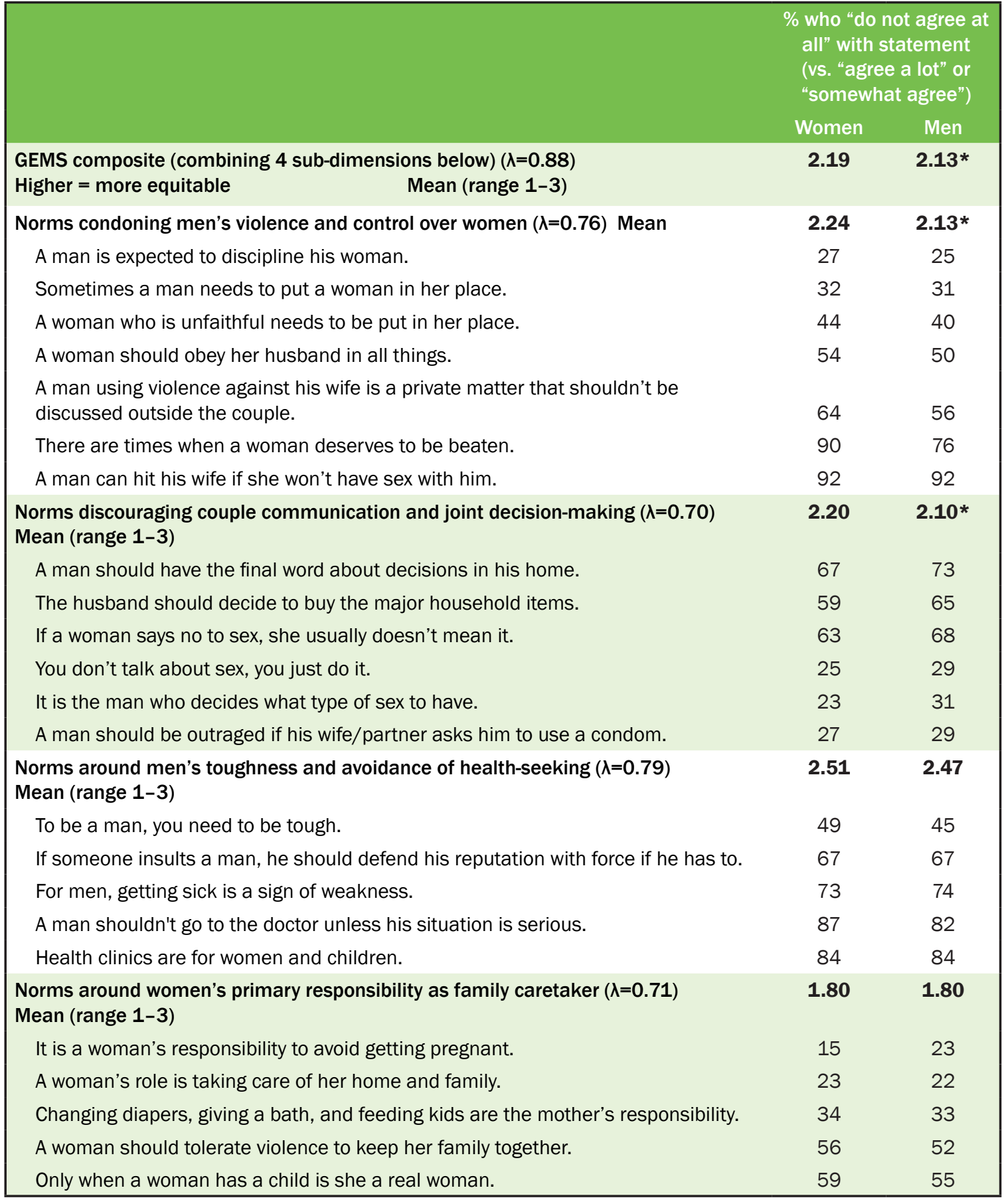

$\lambda=$ Ordinal theta (measure of internal consistency reliability similar to Cronbach's alpha); ${ }^{\star} p<0.001$. $p$-value is for the difference in mean score between women and men, from weighted bivariate analyses that accounted for clustering. Analyses incorporated sampling weights and accounted for clustering.

Both scored more toward the equitable end of the scales (ranging from 2.24 among women for men's violence/control, and 2.10 among men for men as decision-makers in a couple), although we suggest this still represents relatively inequitable norms given the nature of the statements. 
And both had elements with very high prevalence of agreement as well as several questions with low levels of agreement. For violence/control, only one-quarter did not agree that "A man is expected to discipline his woman" and one-third that "Sometimes a man needs to put a woman in her place," while over three-quarters did not condone explicit violence. For norms about men as decision-makers, while three out of six questions, all related to decision-making around sex, were not endorsed by over two-thirds of respondents, the other three questions were not endorsed by a minority of respondents.

Men's and women's responses were quite similar across subdimensions, and while there were some significant differences between women's and men's views on gender norms for some of the scales-men reported significantly more inequitable views than women on the composite GEM Scale, norms regarding men as decision-maker, and norms condoning men's violence and control-these differences were fairly small in magnitude.

To the extent that aggregation of individuals' endorsement of gender equitable norms is indicative of the existence of a norm in the community, these data suggest that there may be some variation in the grip that inequitable norms hold in this population. For example, norms around women's primary responsibility as family caretaker was the most inequitable subdimension (score of 1.80 out of 3 for both males and females) with its elements rarely disagreed with (ranging from 15\% to $59 \%$ not agreeing with individual statements). This suggests a relatively strongly held norm. In contrast, norms around men's toughness and avoidance of health-seeking had the relatively most equitable score out of all the subdimensions (2.51 for females and 2.47 for males), and the highest level of disagreement with individual items. Indeed, for 4 out of 5 of its questions, twothirds or more of participants disagreed, and only about half of participants did not endorse the remaining element (men need to be tough). This may suggest a flexible norm-despite the widely held notion that men's low use of HIV services reflects men's adherence to rigid and restrictive norms that curtail health seeking behavior by men. With respect to the other two subdimensionsmen's violence/control and men as decision-makers in a couple-results suggest that these norms perhaps are beginning to see some erosion of support.

\section{Which dimensions of gender norms matter for HIV testing and treatment outcomes?}

In order to understand the importance of different dimensions of gender norms for improving HIV testing and treatment, we examined whether, and to what extent, each gender norm subdimension was associated with these HIV outcomes (Pulerwitz et al. 2019). We also looked at the association between talking with one's partner about getting an HIV test and HIV testing, since talking with one's partner about getting an HIV test can reflect gender norms and power inequalities in couple communication. Table 4 presents results of multivariate analyses for HIV testing in the last year using the full 27-village baseline data set. Among women, more equitable scores were associated with decreased odds of testing-this finding was significant for the composite GEM Scale (aOR 0.40; 95\% Cl 0.24, 0.68; $p \leq 0.01$ ), norms condoning men's violence (aOR $0.57 ; 95 \% \mathrm{Cl} 0.37,0.88 ; \mathrm{p} \leq 0.05)$, and women's primary responsibility as family caretaker (aOR 0.46; 95\% $\mathrm{Cl} 0.36,0.58 ; \mathrm{p} \leq 0.001$ ). Ancillary analyses (not shown) suggest that this association among women may be explained in part by greater likelihood of testing during pregnancy/after childbirth. Using biological children as a proxy for recent pregnancy (recent 
pregnancy was not asked in the baseline survey), and after controlling for other demographic characteristics, having biological children was significantly associated with both increased odds of testing in the past year $(p<0.01)$ and with support of norms around women's primary responsibility as family caretaker $(p<0.001)$. This also came up in qualitative interviews, as one community member explained, "...if a woman is pregnant...she starts antenatal clinic [and] she does HIV test, for family planning she does HIV test...."

For men there was only one significant association between endorsement of equitable gender norms and HIV testing. Men who scored higher (held more equitable views) regarding men's violence and control over women were less likely to report an HIV test in the past year (aOR 0.65; $95 \% \mathrm{Cl} 0.48,0.90 ; \mathrm{p}<0.05)$. Finally, for both women and men, having talked with their partner about getting tested for HIV increased the odds of getting tested (for women: aOR $1.70 ; 95 \% \mathrm{Cl}$ 1.12, 2.59; $p<0.05$; for men: aOR 1.57; 95\%Cl 1.15, 2.14; $p<0.01)$.

Table 4 Logistic regression results for HIV testing, baseline sample ( $\mathrm{n}=970$ women and $\mathrm{n}=979$ men)

\begin{tabular}{|lcc|}
\hline GEMS (mean score, 23 items) Higher=more equitable & Women & Men \\
aOR & 0.72 \\
Subdimensions & $\mathbf{0 . 4 0 * *}$ & $(0.50,1.05)$ \\
Norms condoning men's violence and control over women & $\mathbf{( 0 . 2 4 , 0 . 6 8 )}$ & $\mathbf{0 . 6 5 *}$ \\
Norms around men as decision-maker in a couple & $\mathbf{0 . 5 7 *}$ & $\mathbf{( 0 . 4 8 , 0 . 9 0 )}$ \\
Norms around men's toughness and avoidance of health-seeking & $\mathbf{( 0 . 3 7 , 0 . 8 8 )}$ & $\mathbf{1 . 0 4}$ \\
Norms around women's primary responsibility as family caretaker & 0.71 & $\mathbf{0 . 8 3 , 1 . 3 0 )}$ \\
Talked with current/most recent sexual partner about getting tested & $(0.50,1.03)$ & $(0.81,1.61)$ \\
for HIV & $\mathbf{0 . 4 6 * * *}$ & 0.81 \\
\hline
\end{tabular}

$* p<0.05, * * p<0.01, * * * p<0.001$; Analyses controlled for age, marital status, and education; incorporated sampling weights; and accounted for clustering.

Turning to HIV treatment, we present results of multivariate regression for current ART use among individuals who reported an HIV-positive status in Table 5. For women, more equitable views on gender norms were significantly associated with increased odds of ART use for the composite GEM Scale (aOR 6.67; 95\% Cl 1.89, 25.00; $p<0.01)$ and three out of four gender subdimensions: men's violence and control (aOR 2.94; 95\% Cl 1.03, 8.33; $p<0.05)$, men as decision-makers in the couple (aOR 5.56; 95\% $\mathrm{Cl} 1.96,14.29 ; \mathrm{p}<0.01)$, and men's toughness and avoidance of health seeking (aOR 2.86; 95\% $\mathrm{Cl} 1.04,7.69 ; \mathrm{p}<0.05$ ). Among men, more equitable views related to men as decision-makers in the couple was significantly associated with increased odds of treatment (aOR $3.57 ; 95 \% \mathrm{Cl} 1.08,12.50 ; \mathrm{p}<0.05)$. The relatively low sample size for these analyses-particularly for men-may have limited the statistical power to identify significant associations in some cases, and likely also contributed to the relatively wide confidence intervals observed. 
Table 5 Logistic regression results for current antiretroviral treatment (ART) use, baseline sample ( $n=122$ women and $n=48$ men)

\begin{tabular}{|c|c|c|}
\hline & $\begin{array}{c}\text { Women } \\
\text { aOR }\end{array}$ & $\begin{array}{l}\text { Men } \\
\text { aOR }\end{array}$ \\
\hline GEM Scale (mean score, 23 items) Higher=more equitable & $\begin{array}{c}6.67 * * \\
(1.89,25.00)\end{array}$ & $\begin{array}{c}1.75 \\
(0.26,12.50)\end{array}$ \\
\hline \multicolumn{3}{|l|}{ Subdimensions } \\
\hline Norms condoning men's violence and control over women & $\begin{array}{c}2.94 * \\
(1.03,8.33)\end{array}$ & $\begin{array}{c}0.94 \\
(0.19,4.76)\end{array}$ \\
\hline Norms around men as decision-maker in a couple & $\begin{array}{c}5.56 * * \\
(1.96,14.29)\end{array}$ & $\begin{array}{c}3.57 * \\
(1.08,12.50)\end{array}$ \\
\hline Norms around men's toughness and avoidance of health-seeking & $\begin{array}{c}2.86 * \\
(1.04,7.69)\end{array}$ & $\begin{array}{c}1.20 \\
(0.24,5.88)\end{array}$ \\
\hline Norms around women's primary responsibility as family caretaker & $\begin{array}{c}1.85 \\
(0.83,4.00)\end{array}$ & $\begin{array}{c}0.77 \\
(0.11,5.26)\end{array}$ \\
\hline
\end{tabular}

$* p<0.05, * * p<0.01, * * * p<0.001$; Analyses controlled for age, marital status, and education; incorporated sampling weights; and accounted for clustering.

\section{EXAMINING SHIFTS IN GENDER NORMS DURING THE INTERVENTION ${ }^{2}$}

Evaluation of the intervention provided further opportunity to examine the process of gender norms change and the pathways through which Tsima may have had an effect. We found that in both treatment and control communities, views on gender norms became significantly more equitable over time among both men and women. Table 6 presents the results of the evaluation for males. Men's composite GEM Scale score increased (became more equitable) by approximately 17 percent from baseline to endline (Adjusted beta 0.33; 95\% Cl 0.25, 0.41; $\mathrm{p} \leq 0.001$ ). There was no significant change over time in intervention versus control communities for the GEM Scale or the subdimensions; thus, the shift toward more equitable views on gender norms cannot be attributed to the intervention.

${ }^{2}$ The data and tables in this section are drawn from Gottert et al. 2020; please use the journal article as primary citation source. 


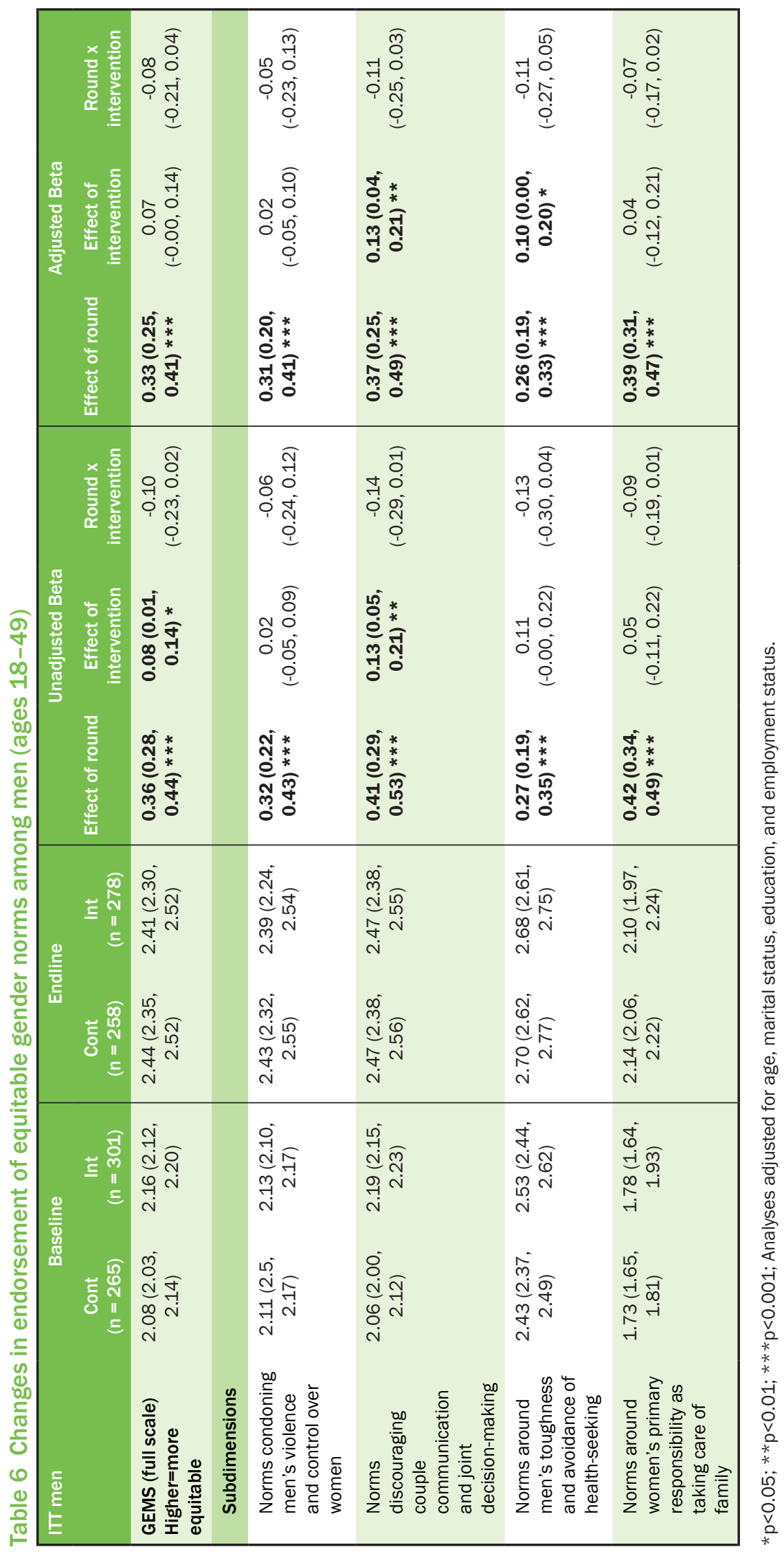


Disaggregating these findings by age, we found that among young men age 18-29, the pattern was similar to that of all men-their views on gender norms became more equitable over time, but there was no difference between the change in intervention and control communities (data not shown). The results of regression analysis for older men are shown in Table 7. Among men ages 30-49, there were differences between intervention and control communities. While older men in intervention communities became more equitable over time, with a composite GEM Scale score increase from 2.16 to 2.40 from baseline to endline, men in control communities had a significantly larger increase, with an increase from 2.07 to 2.51 (Adjusted beta $-0.19 ; 95 \% \mathrm{Cl}$ $-0.32,-0.06 ; p \leq 0.01)$. A similar effect was found with two of the GEM Scale subdimensions (couples communication/joint decision-making and women's role as primary caregiver), with improvements over time among older males significantly greater in control communities than improvements in intervention communities.

Table 8 presents the evaluation results for women's views on gender norms. Women's scores for the composite GEM Scale became more equitable over time, increasing by approximately 13 percent (Adjusted beta $0.25 ; 95 \% \mathrm{Cl} 0.15,0.35 ; p<0.001$ ). Scores on each GEM Scale subdimension also increased significantly (became more equitable) from baseline to endline. As with males however, there were no significant differences between study arms among women; thus, the change in women's gender views cannot be attributed to the intervention. This same finding generally held for both younger and older women (ages 18-29 and 30-49; data not shown). The only exception was that for younger women there was no significant change in views condoning men's violence and control over time, and for older women there was no significant change in views on men's toughness over time. 


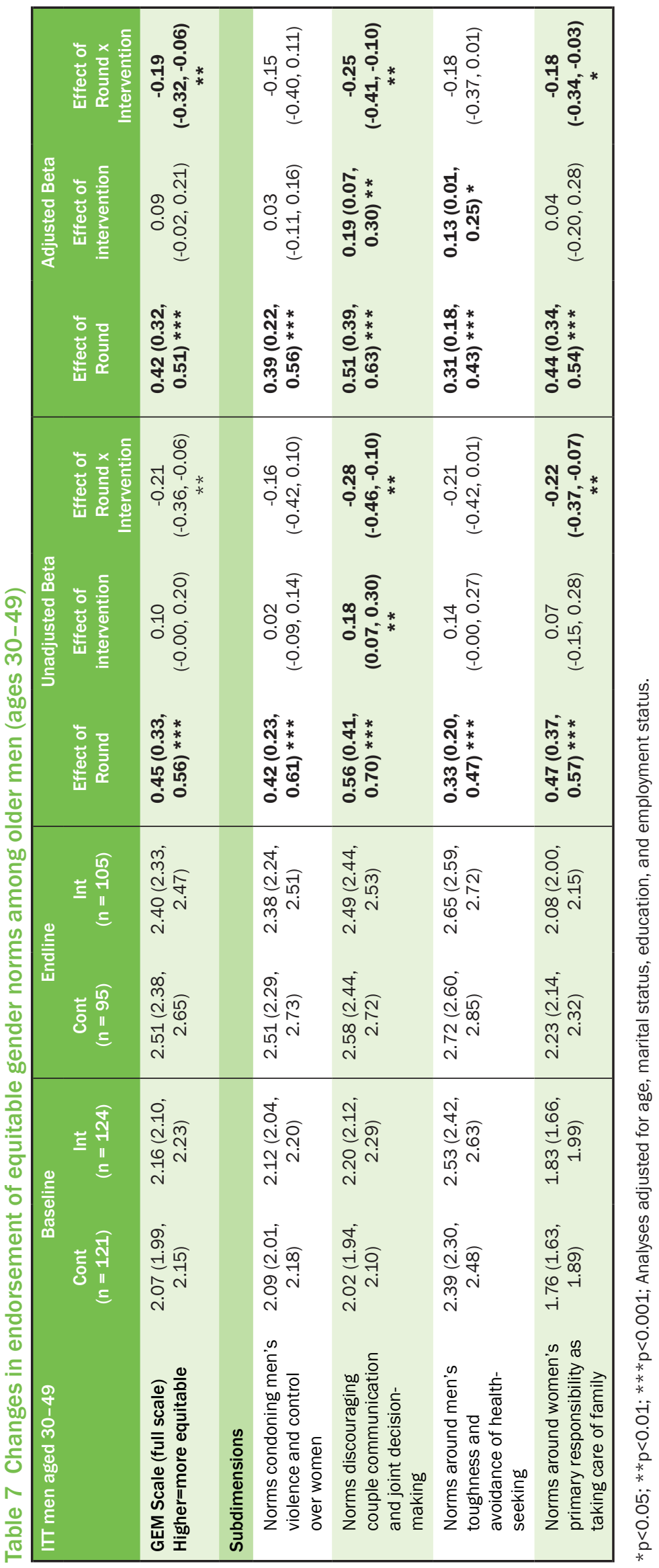




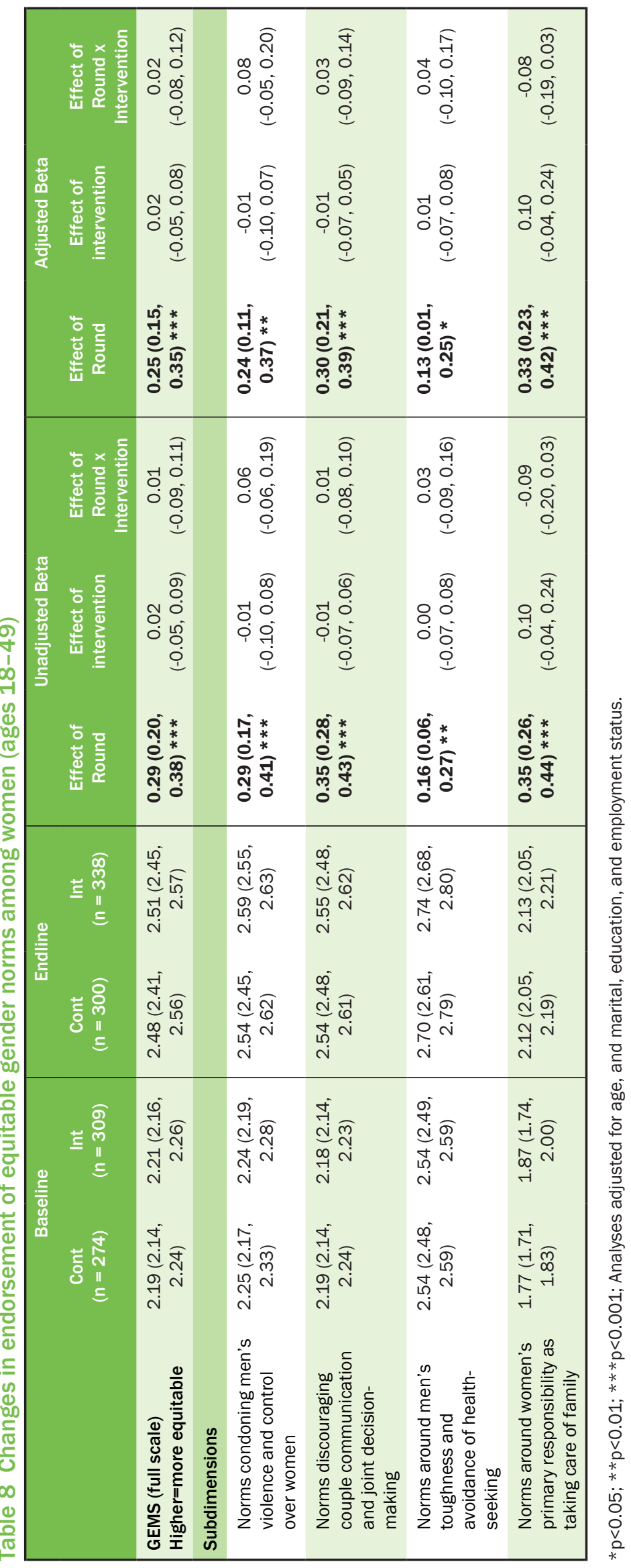


Figure 2 provides a visual representation of the shift in GEM Scale scores toward being more equitable over time, among men and women.

Figure 2 Weighted means of GEMS composite at baseline and endline, for men and women (ages 18-49), stratified by study arm
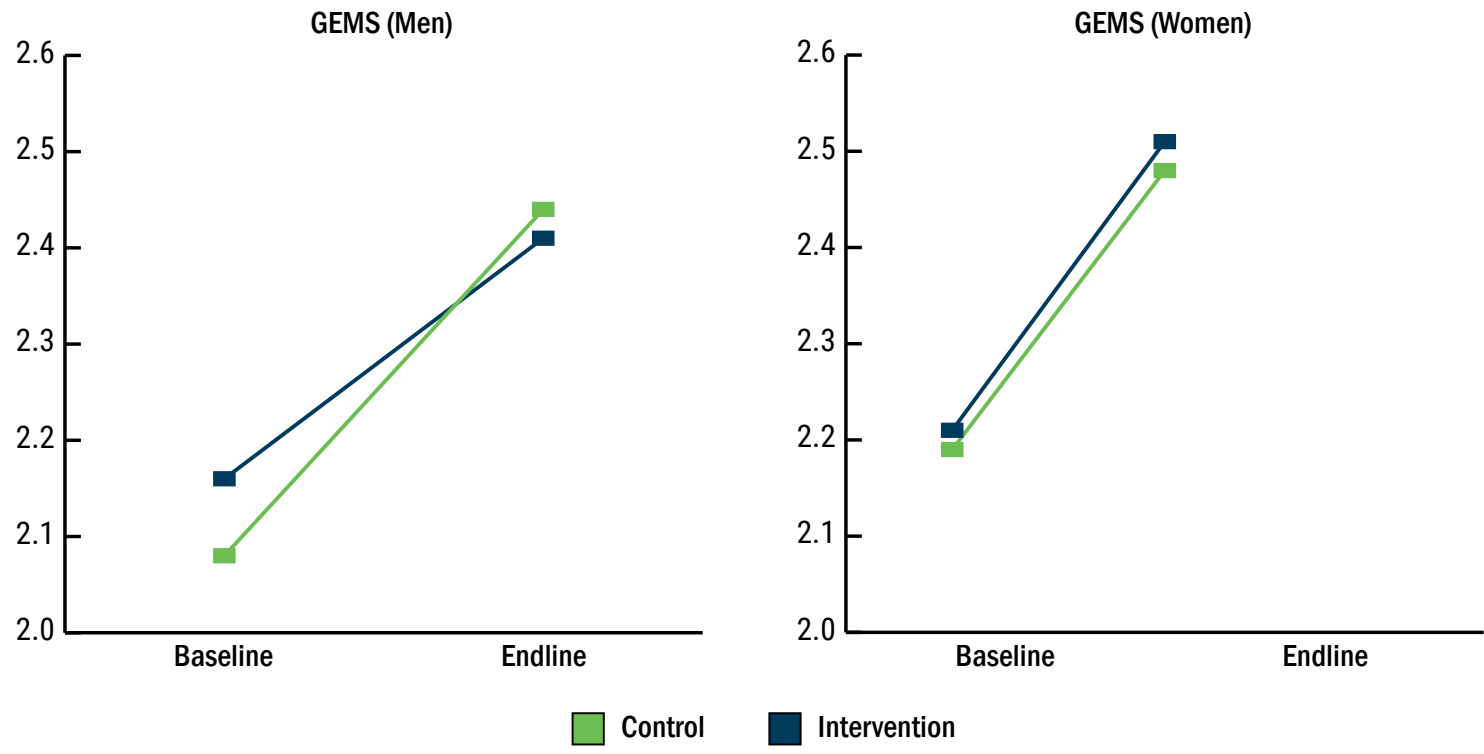

In-depth interviews also reflected a shift toward more equitable views on gender norms in their communities. Many respondents commented on the fact that now people increasingly endorse equitable gender norms. One female community member described the change in the following way:

2 ....previously men wanted to show that they have power even with their wife. Women did all the household duties, it didn't matter if she was tired. A man always wanted to have sex even if a woman was tired...Nowadays we are able to work together, even if they are both coming from work, the husband doesn't mind doing the household duties.

-Female community member

Other dimensions of gender norms were also observed to be changing, with some respondents attributing that change to the intervention:

66 ....many men died because they used to tell themselves that a man must be strong. My sister, if I have something that hurt me inside, I don't keep quiet I speak to my wife about it. If I am sick, I go to the hospital or clinic, I don't want to die. More men died because they didn't want to go to the clinic when they are sick, they keep secrets, because they are believed to be strong and they later have heart attacks. If their relatives die, they do not cry because they are strong, and you can't be strong all the time sometimes you just need to be alone and let your tears out, after that you will be fine. So according to our norms, we grow up knowing that a man must be strong, so Tsima has changed that, if you feel like crying, just let your tears out. Crying heals. 
While, perhaps not surprisingly, community members active in Tsima attributed the change in gender norms to the intervention, the quantitative data showed that the shift toward more equitable gender views was in fact happening across both intervention and control villages. Findings from the third round of qualitative data collection explored what may have been driving this shift across communities. We explored four possible explanations, based on the literature and informal conversations with key informants, including the qualitative field workers who live in the study villages: (1) contamination between intervention and control villages (via direct participation of control village members and/or informal communication about the intervention), (2) other interventions addressing gender norms being conducted in the study area, (3) greater media coverage of gender-based violence (including particularly prominent cases garnering national attention), (4) increasing access to media (TV via satellite dishes; internet via smartphones).

Regarding contamination: while mobilizers in FGDs noted that at times control village participants would participate in activities, survey data demonstrated that such cases were in fact minimal. Instead, informal communication did emerge as prevalent, both in general terms-with control participants often frequenting social venues in intervention villages (and vice versa), and friends and family from different villages spending time together-and specific to sharing of intervention messages, mainly around HIV testing and treatment. While there are administratively distinct villages, many people are related in the area and thus socialize outside village boundaries, making contamination difficult to prevent in something like a cluster randomized trial.

Similarly, as noted above, two-thirds of participants from intervention villages at endline said that Tsima has caused them to talk with others about HIV testing/treatment, and over half said the same about gender or relationships between men and women. Of course, it is unclear whether they talked with control community members-however, given the large extent of inter-village communication generally, this seems a reasonable assumption.

As to whether there were other interventions being implemented in the study area during the three-year study period, very few respondents could think of any, and none explicitly related to gender norms or gender-based violence.

Regarding any increase in gender-based violence-related media coverage: while a number of participants described particularly prominent cases of gender-based violence that had been a focus of news coverage in the last few years, few thought that there was a particularly different level of coverage around these issues than there was four to five years ago (i.e., before the baseline survey), which would be required to explain the shift in norms from baseline to endline.

Finally, participants overwhelmingly confirmed that there has been an increase in access to media in the study area over the last few years. All 26 FGD participants themselves currently own a smartphone and TV ( $n=3$ missing re: TV); about half own a computer. All reported a large increase in the proportion of people in their community who have access to media, and nearly all, when asked, agreed that the increase had begun in earnest within the last four years (i.e., since the trial began). Regarding access to satellite TV, most said that people bought the service ("even if you pay R100 [per month] [approx. \$8 US], there are many channels on that DSTV and more people are using that one," said one male community member). However, over half of 
respondents also said that the government had given either satellite dishes or purchased DSTV: "government has given people DSTV for free, they are busy installing everywhere," said one male community member.

When asked how this increase in media access may have caused changes in gender norms and relationships between men and women, while a few respondents described media's impact as negative, most participants narrated how this has exposed more people to serial dramas ("soapies") and media that model couples communicating and resolving conflicts, and negatively portray IPV.

2 I: Okay, do you think this change [in gender norms] was brought by TV as you mentioned that more people have access to it and DSTV?

$P$ : Yes, there are many changes.

I: Why?

P: People got information from TV or DSTV and got updated about everything that is happening, we are able to watch news and other channels are teaching.

-Male community member

66 ...men are changing because of TV channels, sometimes there is a channel that talk about love...So men start to understand that if "I can change and live in this way, I can be a better person.

-Female community member

66 ...in the television there are different stories of love, you see two people have different views in their relationship you can see them having arguments then later they sit down to resolve their differences and plead for forgiveness to each other....[this] is able to unite two people who have conflicts in their relationship, in that show they end enjoying their relationship again.

-Male community member

I: Okay, could [the change in gender norms] be because people are watching shows that deal with these issues?

P: Yaa that could be the reason because even the issue of women making decision with their partner, we have seen it on TV. ...people discuss about what they see on TV, even here in the tavern people always talk about what is happening in the show Uzalo.

-Male community member 
I think TV and Tsima changed the beliefs of people because on the stories some people act as if they have unhealthy relationship and try to find a doctor who can help them to live a healthy relationship and how they should achieve that and in the process people learn.

-Male community member

\section{UNPACKING GENDER NORM PATHWAYS: INTIMATE PARTNER VIOLENCE $^{3}$}

Analysis of intervention effects (using baseline and endline data from 15 study villages) shows that IPV-both males' reports of perpetration and females' reports of experience of IPV-changed significantly over time, with different patterns by sex and by age. Table $\mathbf{9}$ shows changes in men's reported perpetration of IPV in the past 12 months. Reports of IPV perpetration declined significantly in both intervention and control communities, with men at endline having 60 percent lower odds of reporting IPV perpetration compared with baseline (aOR 0.40; 95\% $\mathrm{Cl} 0.20,0.82$; $\mathrm{p}<0.05)$. However, changes were not significantly different across study arms so this decrease cannot be attributed to the intervention. Disaggregating the data by age (data not shown) showed that younger men (ages 18-29) had even stronger evidence of a decline in IPV perpetration over time (aOR $0.21 ; 95 \% \mathrm{Cl} 0.10,0.44 ; \mathrm{p}<0.001$ ) (data not shown), though the change was still not attributable to the intervention. In contrast, there was no significant change in reported IPV perpetration among older men (ages 30-49) over time or between study arms.

Women's experience of IPV increased in control communities and decreased in intervention communities (Table 10). At endline, in intervention communities, women (ages 18-49) had half the chance of experiencing IPV than women in control communities (aOR 0.53; 95\% $\mathrm{Cl} 0.31,0.89$; $\mathrm{p} \leq 0.05)$, a significant intervention effect. This same pattern was observed for younger women (ages 18-29). In contrast, among older women (ages 30-49), there was an overall doubling of risk of experiencing IPV over time; there was no intervention effect (Table 11).

\footnotetext{
${ }^{3}$ The data and tables in this section are drawn from Gottert et al. 2020; please use the journal article as primary citation source.
} 

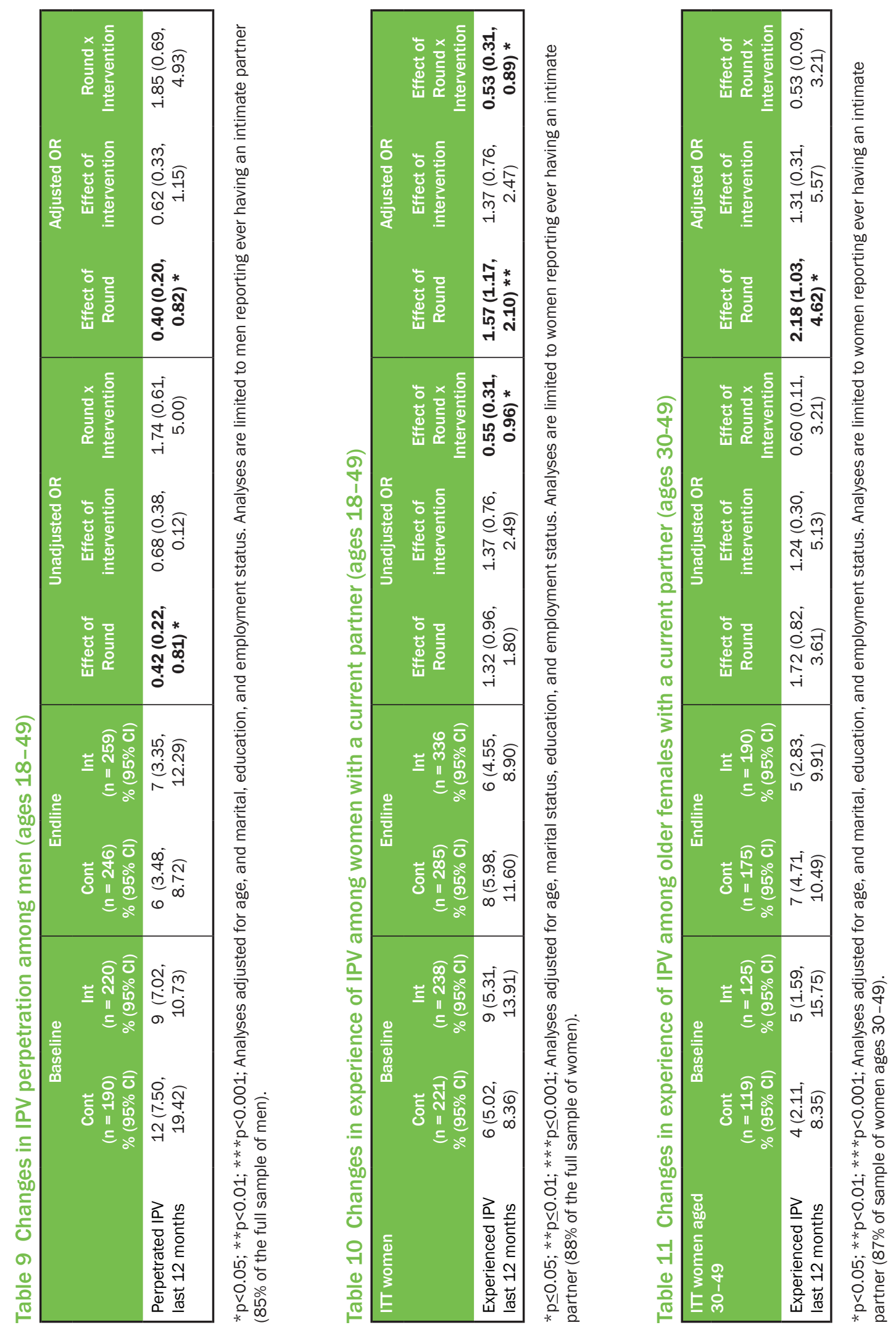
Figure 3 Weighted percentage of IPV at baseline and endline, for men and women (ages 18-49), stratified by study arm

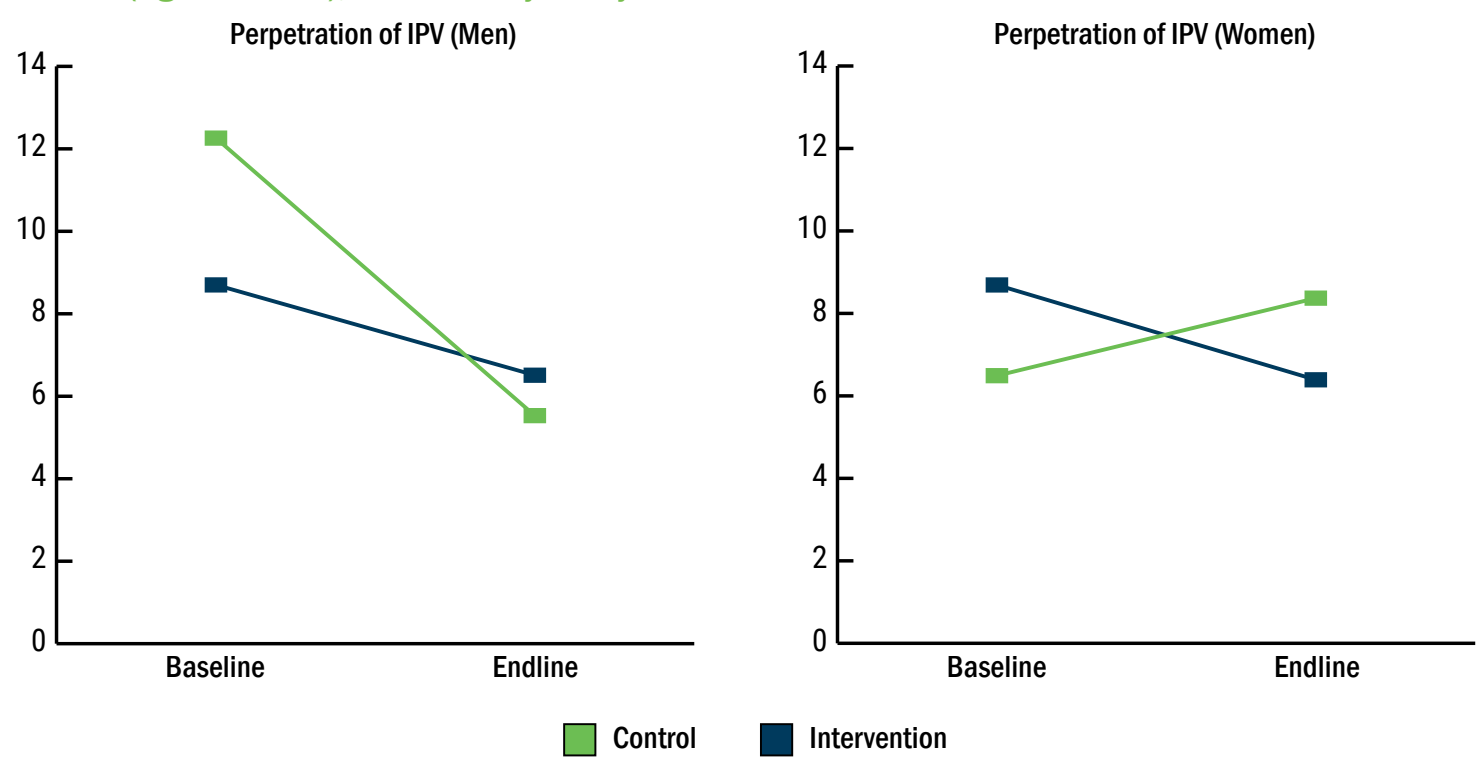

In-depth interviews with women and men in intervention communities also explored IPV. ${ }^{4}$ Almost all participants who discussed IPV described decreases in IPV perpetration over time, reinforcing the quantitative findings. How this decline in IPV was happening was attributed in part to decreasing acceptability of violence against women, as well as to couples learning to communicate more constructively with each other as a result of Tsima. For example, one man explained what he learned from Tsima that helped him both understand that violence against women is wrong and the concrete skills he needed to stop perpetrating violence:

66 Tsima helped me to understand how to treat a woman...[my partner and I] are very close.... According to what I have learned in Tsima...we must always communicate with each other. So Tsima helped me. I was not communicating with her, if I wanted to do something I was doing it without communicating with her. She was always complaining about it, arguing and sometimes I was abusing her physically when she complained. But Tsima has changed that, we always communicate nowadays.

-Male community member

Similarly, a mobilizer related the following story:

There is someone I know, he was always fighting with his partner because there was no communication. His wife was afraid of him, if she needed something from her husband she would not say anything because her husband was always shouting at her and abusing her physically. So the husband attended a [Tsima] workshop and he was asking many questions, he wanted to understand some of the things [about couple communication]. The following day of the workshop he came with his wife, they attended together. After some few days, I saw them going to town and they were happy. [Usually] the

${ }^{4}$ The qualitative analysis in this section draws from Leddy et al. (in preparation). 
husband was the one who was buying groceries for the family, but now the wife is the one who buys the groceries. So there is a big change, they are always happy because of Tsima.

-Female mobilizer

In addition, in line with the effects of increasing access to media on gender norms, described by respondents in post-endline interviews, such change was also articulated as influencing couple conflict and relationship violence.

2 I always watch those soaps, like "Bokithila" and "Nyaninyani" (TV shows), it helps people to make decision if there is abuse that it is happening in their marriage or in their relationship. It is either you come out or get help, or divorce or leave the relationship or stay and die in that relationship.... There is another soapie...they are just talking about violence or unhealthy relationships.... At the end they provide counselling to these couples, teaching them until they have a solution or understand.

-Female community member

\section{UNPACKING GENDER NORM PATHWAYS: HIV TESTING AND TREATMENT}

Note: The main trial findings (study funded by NIH) were presented at IAS in 2020 (Lippman et al. 2020) and will be available in publication shortly. Main study findings are not covered in this report, however links to published findings will be posted here when they become available. In general, quantitative data showed improvements in testing across both intervention and control communities over time, with evidence of greater increases in the intervention communities, demonstrating an intervention effect. Similarly, retention in care was significantly higher among women in the intervention communities as compared to control communities and overall the 90-90-90 indicators at the end of the study period demonstrated higher rates of known status (men and women), ART initiation (women), and viral suppression (men) in intervention villages as compared to control villages (Lippman et al. forthcoming).

Qualitative data shed light on pathways through which changes in gender norms and IPV may influence testing and treatment uptake. ${ }^{5}$ Some respondents noted that Tsima improved norms around equitable couple communication and promoted skill-building around constructive communication, and that this facilitated HIV status disclosure and HIV treatment retention:

\footnotetext{
2 For those who are attending Tsima there is a change, they are communicating, and have trust in their relationships. I will give an example about a man who attended a support group... He told us that his wife did not trust him and there was no communication with his wife. But Tsima helped him. Now he is trusted by his wife and they always communicate. He disclosed his status to his wife and children, [and they] remind him to take treatment at seven o'clock in the evenings.
}

-Female community member

${ }^{5}$ The qualitative analysis in this section draws from Leddy et al (in preparation). 
Many participants also articulated an association of better communication between partners on reducing IPV, which in turn facilitates HIV treatment uptake and retention:

There is change in terms of violence, because there was violence that was

happening in the household. And some of the people were even afraid to take treatment, they were always hiding because of the violence that was happening in the household. They were afraid that, they might lose marriages but since Tsima came, there is a communication in relationships, there is no violence. People are taking treatment, and continue to live.

-Female CAT member

Participants also described how changed gender norms could impact HIV testing and HIV status disclosure. The examples given were often in regard to norms that our survey findings showed were more tenuously held, such as men's toughness and avoidance of health-seeking. For example:

There is a change because nowadays men are sharing when they have problem, the issue of being brave according to the culture it has changed since they [engaged] in Tsima. Men are now doing HIV testing, they have taken action on that, and they also disclosing their HIV status they also volunteer to break the silence, they are telling their friends and disclosing HIV status.

-Female mobilizer

Finally, increased awareness about the benefits of early testing and treatment, as well as treatment as prevention, was described as intersecting with gender norms in ways that facilitated uptake of HIV services among men. Specifically, learning that if taken early enough, treatment can allow you to live a full and productive life, never look sick, and eliminate the possibility of transmitting to your sexual partners, appeared to alleviate concerns that were founded in meeting expectations of themselves as men, making this less of an obstacle to accessing services. This may have been particularly important for a subset of men who appeared resistant to testing as a result of these concerns. One man articulated this in detail:

66 If it wasn't for Tsima I don't think I would have tested. I was scared that if I go to the clinic and get tested and find that I'm HIV-positive I will be confronted with stress, but the mobilizer came and taught us the importance of knowing my HIV status and not to be scared. It does not help to know your status when you are critically ill or a bed ridden, so it's good to know your status while you are walking on your feet because it will be very easy to recover on time and even people cannot notice that you are sick...So it has helped me because I managed to know my status and I have also taken my partner with me to get tested though I was scared, so today we are no longer scared. Each and every two months I make sure that I go to the clinic to recheck my status because it might happen things changed at any time...My wife can fall pregnant, if she knows that she is HIV-positive our infant will be protected. So that is why I say Tsima has managed to help in such things like that... have been encouraged by the mobilizer from Tsima that if you test you don't protect yourself only, like I said that I now I'm planning to have another child.

-Male community member 


\section{COST ANALYSIS}

\section{Costs}

Table 12 shows the total intervention costs by main cost category. The total cost of the intervention over the three-year period was US $\$ 429,000$ (US $\$ 143,000$ per year). While we are not aware of other cost analyses of community mobilization for HIV prevention and treatment among general populations, the overall annual cost of the project is similar to a community mobilization project to prevent IPV in Uganda (US\$139,000 per year) (Michaels-lgbokwe et al. 2017). Mobilization activities accounted for 77 percent of the total costs at US $\$ 328,000$ (US $\$ 109,000$ ). Supervision accounted for 15 percent of costs-US $\$ 63,000$ (US $\$ 21,000$ ). Across categories, the largest cost driver was personnel, in total accounting for 80 percent of intervention costs.

Table 12 Costs of the Tsima intervention (US\$, 2018)

\begin{tabular}{|c|c|c|c|}
\hline & $\begin{array}{l}\text { Total 3-year } \\
\text { costs }\end{array}$ & $\begin{array}{c}\text { Total annual } \\
\text { costs }\end{array}$ & $\begin{array}{c}\% \\
\text { costs }\end{array}$ \\
\hline \multicolumn{4}{|l|}{ Start-up } \\
\hline Personnel & $26,777.07$ & $8,925.69$ & \\
\hline Operating costs & $2,471.60$ & 823.87 & \\
\hline Other costs & $2,339.89$ & 779.96 & \\
\hline Sub-total & $31,588.56$ & $10,529.52$ & 7 \\
\hline \multicolumn{4}{|l|}{ Supervision } \\
\hline Personnel & $53,696.01$ & $17,898.67$ & \\
\hline Transport/vehicle operating costs & $7,686.12$ & $2,562.04$ & \\
\hline Share of overhead & $1,772.30$ & 590.77 & \\
\hline Sub-total & $63,154.43$ & $21,051.48$ & 15 \\
\hline \multicolumn{4}{|l|}{ Training } \\
\hline CAT training & $1,090.91$ & 363.64 & \\
\hline Skills training & $3,073.02$ & $1,024.34$ & \\
\hline Share of overhead & $1,772.30$ & 590.77 & \\
\hline Sub-total & $5,936.23$ & $1,978.74$ & 1 \\
\hline \multicolumn{4}{|l|}{ Mobilization activities } \\
\hline Personnel & $262,149.03$ & $87,383.01$ & \\
\hline Workshops & $40,878.22$ & $13,626.07$ & \\
\hline Events & $8,449.71$ & $2,816.57$ & \\
\hline Outreach and small groups & $6,993.77$ & $2,331.26$ & \\
\hline Transport and catering & $3,394.26$ & $1,131.42$ & \\
\hline Branding & $2,188.08$ & 729.36 & \\
\hline Communication & $2,842.75$ & 947.58 & \\
\hline Share of overhead & $1,772.30$ & 590.77 & \\
\hline Sub-total & $328,668.12$ & $109,556.04$ & 77 \\
\hline Total costs & $429,347.34$ & $143,115.78$ & 100 \\
\hline
\end{tabular}




\section{Outcomes}

Table 13 shows attendance by village. There was a total of 91,704 contacts in the period 2015 to 2018 , averaging about 30,000 contacts per year.

Table 13 Total attendance (number of contacts) at Tsima events (2015-2018)

\begin{tabular}{|lrrrr|}
\hline Village & Year 1 & \multicolumn{1}{c}{ Year 2 } & \multicolumn{1}{c|}{ Year 3 } & \multicolumn{1}{c|}{ Total } \\
Belfast & 4,253 & 4,843 & 4,250 & 13,346 \\
Dumphries C & 3,076 & 3,005 & 3,185 & 9,266 \\
Huntington & 5,015 & 5,807 & 5,203 & 16,025 \\
Ireagh B & 2,548 & 2,478 & 2,633 & 7,659 \\
Lillydale & 3,560 & 3,299 & 3,232 & 10,091 \\
MP Stream & 4,397 & 4,554 & 3,529 & 12,480 \\
Makaringe & 3,914 & 4,418 & 3,979 & 12,311 \\
Rolle C & 3,641 & 3,521 & 3,364 & 10,526 \\
Total & 30,404 & 31,925 & 29,375 & 91,704 \\
\hline
\end{tabular}

Attendance by activity type is shown in

Table 14. There were 78,000 contacts, the vast majority participating through outreach activities.
Table 14 Total attendance by activity (2015-2018)

\begin{tabular}{|lc|}
\hline Activity & Contacts \\
\hline Outreach & 76,473 \\
Events & 2,160 \\
Workshops & 13,071 \\
Total & 91,704 \\
\hline
\end{tabular}

\section{Average costs}

The cost per contact (attendance) was estimated to be US $\$ 4.68$. The costs per contact by activity type are shown in Table 15. The average cost for outreach activities was US $\$ 2.38$, compared to US $\$ 47.04$ for events and US\$11.17 for workshops.

Table 15 Average costs of each activity type

\begin{tabular}{|lccc|}
\hline Activity & Contacts (No.) & Costs (US\$) & Average costs (US\$) \\
Outreach & 76,473 & $81,733.45$ & 2.38 \\
Events & 2,160 & $101,603.25$ & 47.04 \\
Workshops & 13,071 & $146,010.64$ & 11.17 \\
Any activity & 91,704 & $429,347.34$ & 4.68 \\
\hline
\end{tabular}

Note: Included in each activity cost is a share of start-up, supervision, overhead, and training costs 


\section{DISCUSSION}

This study addressed key gaps in the field around understanding the influence of different gender norms on HIV testing and treatment, as well as elucidating the process by which gender norms change and the ways in which this change may lead to improved HIV service uptake and reduced violence and HIV risk.

We explored four subdimensions of gender norms hypothesized to be related to HIV service uptake: norms condoning men's violence and control over women, men as decision-maker in a couple, men's toughness and avoidance of health-seeking, and women's primary responsibility as family caretaker. We found variation in the baseline quantitative data in both how strongly (where on the scale of inequitable to equitable people's average score lay) and how widely (the percent of respondents agreeing with each statement of the subscale) these norms were held. We found that norms around women's primary responsibility as family caretaker were the least equitable (i.e., most endorsed). Norms around men's toughness and avoidance of help seeking were the most equitable, and thus we should be open to other potential explanations for men's low use of HIV services such as clinic-related barriers. The other two subdimensions (men's violence/ control and men's role as decision-makers) had more middle-of-range scores. Specifically, scale items regarding men's explicit use of violence against women, and decision-making around sex, had low levels of endorsement, whereas others-particularly those relating to men's control over women-had quite high levels of endorsement. The more varied views on these subdimensions may suggest that they are beginning to shift.

While the subdimensions of gender norms were endorsed by similar proportions of women and men, how these different gender norms may influence HIV testing and treatment differed between them. Among women, somewhat surprisingly, equitable views on gender norms-the composite GEM Scale scores, and particularly the subdimension of women's responsibility as family caretaker-were associated with a decreased odds of testing. Ancillary analyses, as well as the qualitative data, suggest that among women, this association may be due to women being more likely to endorse the norms around women being the main family caretaker during their active childbearing years, which is also the time they are likeliest to be tested for HIV (i.e., during pregnancy). Among men, the overall GEM Scale did not show an association with HIV testing, but more equitable scores for the subdimension of men's violence and control over women was significantly associated with decreased odds of testing. Reasons for this association for men remain unclear and merit further exploration.

In line with our expectations, women living with HIV who held more equitable views about gender norms were significantly more likely to be using ART, a finding reflected in previous qualitative research (Ahmed et al. 2018). This was true for the composite GEM Scale scores and all subdimensions except for women's primary responsibility as family caretaker. Men living with HIV who held equitable views about men's role as decision-maker in a couple were significantly more likely to be on HIV treatment. Thus, for women in this baseline sample, views toward gender norms were significantly associated with HIV testing and treatment use, yet operate in different 
ways for HIV testing (more equitable linked with less testing) than for HIV treatment (more equitable linked with more ART use). It could be that women who hold more equitable views are in more trusting relationships and perceive less risk for HIV, and therefore are less likely to get tested. For male respondents these associations were also seen for norms regarding men as decision-makers and men's violence and control. (Note that we might have seen more statistically significant associations had our sample of men who reported living with HIV been larger.)

The evaluation analyses clearly demonstrated that views toward gender norms can and do change, among both women and men. We found significant and large-scale increases from baseline to endline in support of equitable gender norms across intervention and control communities. Thus, no effect of the intervention was observed on gender norms. Qualitative data suggest that the broad shift may have been driven by rapid escalation in media access across the study area, providing many more people with access to modeling of more equitable gender norms and conflict resolution in relationships, as well as negative portrayals of partner violence. Many "soapies" were described as frequently depicting more equitable couple communication and negatively portraying violence through their narratives; talk shows were also mentioned as highlighting these topics. As there was no differentiation in television programming between intervention and control communities, it appeared that increased accessibility to mediaincluding via the government's installation of satellite dishes across the area-was key. We asked specifically about two "edutainment" TV serial dramas that focus directly on gender norms, IPV, and HIV: Soul City and MTV's Shuga Down Under (South African adaptation of Shuga). While most participants had heard of and liked Soul City, they also noted that it has been on for many years and that they themselves didn't watch it as much in the last few years. None of the participants we interviewed had heard of MTV Shuga. This finding highlights the critical need to understand societal-level shifts, especially in TV/internet access, and how these shifts can be leveraged for health behavior change. (Note that since Tsima was evaluated via a community RCT, media strategies were not employed since this would have reached both intervention and control communities.)

While the shift to more support for equitable norms was observed for the composite GEM Scale and all four subdimensions and showed similar rates of improvement among females and males, there were differences by age. We found that while older men in intervention communities did become more equitable, they did not change their views as much as men in control communities on two of the four subdimensions. Older men also had less exposure to Tsima than younger men.

Inequitable gender norms have been shown to both perpetuate and be reinforced by IPV. Indeed, the intervention did lead to significant intervention effects in reducing women's reported experience of IPV, where women in the intervention communities reported reductions while women in the control communities did not. Among men, reports of IPV perpetration decreased significantly from baseline to endline. However, as with the changes in views toward gender norms, the decrease in men's reports of IPV perpetration occurred in both study arms, and thus there was no clear intervention effect. Thus, while in intervention communities women's reports of decreased experience of IPV align with men's reports of decreased perpetration, women's and men's reports of IPV in control communities are at odds, with men reporting decreased IPV perpetration and women reporting increased IPV experience. It may be that social desirability bias among males in control communities led to underreporting of IPV perpetration given the shifts to 
lower endorsement of norms around men's violence and control. While a recent study of couples in Tanzania concluded that social desirability bias was not a major factor in discordant reports of IPV, it was a cross-sectional study in a setting where justification of violence against women was high (Halim et al. 2018).

Further, disaggregating IPV data by age showed that older men did not report a decrease in IPV perpetration over time, and older women reported a significant increase over time. It could be that older men are more resistant to changing established patterns of behavior or are more set in their ways, and older women not only experience older men's unchanged IPV perpetration, but at the same time have a better understanding of what constitutes IPV and thus increased their reporting.

In general, quantitative data from the Clinic Link system showed improvements in testing across both intervention and control communities, but a higher rate of improvement in intervention communities, demonstrating evidence of an intervention effect (Lippman et al. forthcoming). It is possible that decreases in IPV could be a path to testing for women and men. Qualitative data suggest that there were diverse pathways through which changes in gender norms may have had an effect on IPV and HIV testing and treatment. These included: 1) There were important changes in gender norms, IPV, and HIV testing, treatment, and disclosure over the study period; 2) Tsima encouraged and built skills around more equitable, constructive communication between intimate partners; 3 ) these shifts helped men find alternatives to violence and facilitated treatment uptake and retention; and 4) new knowledge about the benefits of treatment as prevention was more actionable, for example, because of reduced endorsement of inequitable norms regarding men's health seeking and men's toughness.

Broad-based shifts in gender norms were observed across the intervention and control sites, and the qualitative data suggest that these changes could be fueled by increased media exposure. In intervention communities only, Tsima mobilization activities aimed to foster critical thinking about power relations, violence, and inequity, as well as to build skills around couple communication and conflict resolution. It may be that the norms around IPV may have shifted in both intervention and control communities due to media exposure, but only in Tsima communities did it translate to changed behavior due to the additional skill-building activities.

Another way in which Tsima may have had its effects again has to do with the gender norms change process. In Tsima communities, more equitable views on gender norms enabled acting on new knowledge that individuals may or may not have been able to pursue without the gender norms shift. Specifically, Tsima participants learned about the benefits of early testing and treatment and treatment as prevention-this knowledge, together with views that no longer adhered so tightly with norms discouraging male health care seeking, may have facilitated men's ability to seek testing services.

The study found notable differences by age, particularly among males. Older men were less likely to participate in Tsima, and, while their views on gender became more equitable over time, men in intervention communities reported smaller increases in gender scale scores than men in control communities. Further, older men did not reduce their perpetration of IPV. Why this might be the case is an area for further research. Findings from the multi-country IMAGES study found 
that in some settings younger men were more likely than older men to have gender equitable attitudes, in other settings it was the older men who expressed more equitable views, and in others there was no significant difference by age (Levtov et al. 2014).

Finally, the cost analysis estimates total program costs of US $\$ 429,000$ for the three-year period 2015-2018, and the associated average cost was US $\$ 4.68$ per contact. After isolating the mobilization activities, we estimated total costs (and average costs) of US $\$ 182,000$ (US\$2.38) for outreach, US $\$ 102,000$ (US $\$ 47.04$ ) for events, and US $\$ 146,000$ (US\$11.17) for workshop activities. Events and workshops were more expensive because workshops included the cost of food and beverages (often lunch, a snack, and drinks), and events included more expensive items such as renting audio visual equipment, equipment to wash cars with, and tent rentals.

\section{Limitations}

We note several limitations to the study. First, the survey data are based on self-reported responses, which may be subject to social desirability bias as well as misreporting due to confusion around question meaning (Mooney et al. 2018). However, we note that some of the potential sources of bias (e.g., being asked sensitive questions in general) are mitigated by having a control group, where the bias would be seen in both groups, and thus seeing differences in responses already takes this potential bias into account. Moreover, some evidence suggests that studies that have explicitly controlled for social desirability do not tend to find that it has a significant impact on the interpretation of study results (Latkin, Vlahov, \& Anthony 1993). Second, there were a few statistically significant differences in sociodemographic characteristics between respondents at baseline and endline, and between intervention and control participants at baseline or endline; however, we controlled for these characteristics in all regression analyses. Third, the small reported number of HIV-positive individuals among our survey respondents, particularly among men, meant that we may not have had adequate statistical power for inferential analyses for treatment-related outcomes. Finally, for tests of associations presented in this report, due to the number of tests, there is a potential for increased detection of significant associations, when in fact they may be due to chance. 


\section{CONCLUSION AND RECOMMENDATIONS}

Taken together, findings from this study-implemented in the context of a rigorous RCTdemonstrated that shifting norms to be more gender-equitable can create an enabling environment for significant decreases in IPV as well as pathways toward improved uptake of HIV treatment. Further, decreases in IPV could be a path to testing for women and men. The study also raised questions about the extent to which men's low use of HIV services is explained by gender norms in this setting. The study demonstrated that shifts in views toward gender can be fostered in a meaningful way at the community level, including via burgeoning social changes that are often overlooked in studies about health-related beliefs and behaviors. Such shifts in gender norms may also provide an important boost at the individual level. To apply these results most effectively to programs, we recommend:

1. When implementing gender transformative work to address HIV and IPV, conduct rigorous research to determine the extent to which different gender norms influence what-and whosebehavior, and tailor interventions accordingly. If such diagnostic research is not possible, examine relevant national/subnational studies or surveys that can provide insight into these dynamics.

2. Combine activities to shift gender norms with skill building to ensure translation into practice, such as more equitable couple communication and conflict negotiation as a way to prevent IPV and encourage HIV testing and treatment.

3. Simultaneously address gender-related barriers to HIV service use-such as gender norms and IPV that prevent disclosure and treatment seeking-while providing concrete information about the benefits of early testing and treatment and TasP to ensure informed decision-making about health and to maximize impact on HIV testing and treatment.

4. When certain gender norms are shown not to be a predominant barrier to the use of HIV services for a subpopulation, explore and address other possible impediments to service utilization, such as clinic hours, wait time, concerns about confidentiality, etc., some of which can be gendered issues, but not always.

5. Conduct additional rigorous evaluation studies designed to explicitly explore implementation questions, including optimal exposure and length of interventions to change gender norms, efficient combinations of intervention components, etc. There are a limited number of studies that have explicitly assessed these issues, but the evidence base is growing. For example, SOAR's Community Responses study, which evaluates a community-based HIV and sexual and gender-based violence prevention program, is looking at the question of optimal exposure.

6. Add costing components to more studies to build the body of costing information in order to inform intervention design, implementation, and scale up.

7. Follow up participants over the longer term to observe possible additional/ripple effects of interventions like Tsima on, for example, new HIV infections and retention in care. 
8. Pay greater attention in research, strategic planning, and intervention development to critical, broader, social changes underway in a program's setting-including rapidly expanding media and internet access across sub-Saharan Africa-and how these changes can be leveraged to improve health behaviors. 


\section{REFERENCES}

Ahmed, Shahira et al. 2018. "Why do people living with HIV not initiate treatment? A systematic review of qualitative evidence from low- and middle-income countries," Social Science \& Medicine 213: 72-84. doi: 10.1016/j. socscimed.2018.05.048

Cohen, M.S., M. McCauley, and T. Gamble. 2012. "HIV treatment as prevention and HPTN 052," Current Opinion in HIV and AIDS 7(2): 99-105. doi: 10.1097/COH.0b013e32834f5cf2

Cohen, M.S. et al. 2016. "Antiretroviral therapy for the prevention of HIV-1 transmission," New England Journal of Medicine 375(9): 830-9. doi: 10.1056/NEJMoa1600693

Dunkle, K.L. et al. 2006. "Perpetration of partner violence and HIV risk behaviour among young men in the rural Eastern Cape, South Africa,” AIDS 20(16): 2107-14. doi: 10.1097/01.aids.0000247582.00826.52

Dworkin, S.L., S. Treves-Kagan, and S.A. Lippman. 2013. “Gender-transformative interventions to reduce HIV risks and violence with heterosexually-active men: a review of the global evidence," AIDS and Behavior 17(9): 2845-2863. doi: 10.1007/s10461-013-0565-2

GHHC. 2018. "Reference case for estimating the costs of global health services and interventions," Internet. Available: https://ghcosting.org/pages/standards/reference_case

Gottert, A. et al. 2016. “Measuring men's gender norms and gender role conflict/stress in a high HIV-prevalence South African setting," AIDS and Behavior 20(8): 1785-95. doi: 10.1007/s10461-016-1374-1

Gottert, A., et al. 2017. "Gender norms, gender role conflict/stress and HIV risk behaviors among men in Mpumalanga, South Africa," AIDS and Behavior 22(6): 1858-1869. doi: 10.1007/s10461-017-1706-9

Gottert, A. et al. 2020. "Gaining traction: Promising shifts in gender norms and intimate partner violence in the context of a community-based HIV prevention trial in South Africa," PLoS One 15(8): e0237084. doi: 10.1371/journal. pone.0237084

Grinsztejn, B. et al. 2014. "Effects of early versus delayed initiation of antiretroviral treatment on clinical outcomes of HIV-1 infection: results from the phase 3 HPTN 052 randomised controlled trial," The Lancet Infectious Diseases 14(4): 281-90. doi: 10.1016/S1473-3099(13)70692-3

Haberland, N. 2015. "The case for addressing gender and power in sexuality and HIV education: A comprehensive review of evaluation studies," International Perspectives on Sexual and Reproductive Health 41(1): 31-42. doi: $10.1363 / 4103115$

Halim, N. 2018. "Variability and validity of intimate partner violence reporting by couples in Tanzania," PLoS One 13(3): e0193253. doi: 10.1371/journal.pone.0193253.

Hatcher AM et al. 2015. "Intimate partner violence and engagement in HIV care and treatment among women: a systematic review and meta-analysis," AIDS 29(16): 2183-94.

Human Sciences Research Council (HSRC). 2018. The fifth South African National HIV prevalence, incidence, behaviour and communication survey, 2017.

The INSIGHT START Study Group. 2015. "Initiation of antiretroviral therapy in early asymptomatic HIV infection," New England Journal of Medicine 373(9): 795-807. doi: 10.1056/NEJMoa1506816

Jewkes, R.K. et al. 2010. "Intimate partner violence, relationship power inequity, and incidence of HIV infection in young women in South Africa: a cohort study," The Lancet 376(9734): 41-8. doi: 10.1016/S0140-6736(10)60548-X

Kahn, K. et al. 2012. "Profile: Agincourt health and socio-demographic surveillance system," International Journal of Epidemiology 41(4): 988-1001. doi: 10.1093/ije/dys115

Latkin, C.A., C. Vlahov, and J.C. Anthony. 1993. "Socially desirable responding and self-reported HIV infection risk behaviors among intravenous drug users,” Addiction. 88(4): 517-26. doi: 10.1111/j.1360-0443.1993.tb02058.x

Leddy A et al. In preparation: Delineating the process of gender norms change and pathways to HIV service uptake: Evidence from a community mobilization intervention in South Africa

Levtov, R. et al. 2014. "Pathways to gender-equitable men: Findings from the International Men and Gender Equality Survey in eight countries," Men and Masculinities 17(5): 467-501. doi: 10.1177/1097184X14558234 
Lippman, S.A. et al. 2017a. "Evaluation of the Tsima community mobilization intervention to improve engagement in HIV testing and care in South Africa: study protocol for a cluster randomized trial," Implementation Science 12(1): 9. doi: 10.1186/s13012-016-0541-0

Lippman, S.A. et al. 2017b. "Community mobilization for HIV testing uptake: Results from a community randomized trial of a theory-based intervention in rural South Africa," Journal of Acquired Immune Deficiency Syndromes 74(Suppl 1): S44-S51. doi: 10.1097/QAI.0000000000001207

Lippman, S.A. et al. 2020. "Community mobilization to improve engagement in HIV testing, linkage to care and retention in care in 15 villages in South Africa: The Tsima cluster-randomized controlled trial." Oral presentation, 23rd International AIDS Conference, 6-10 July 2020.

Michaels-Igbokwe, Christine et al. 2016. "Cost and cost-effectiveness analysis of community mobilisation intervention to reduce intimate partner violence in Kampala, Uganda," BMC Public Health 16: 196. doi: 10.1186/s12889-016-2883-6

Montaner, J. et al. 2014. "Expansion of HAART coverage is associated with sustained decreases in HIV/AIDS morbidity, mortality and HIV transmission: the 'HIV treatment as prevention' experience in a Canadian setting," PLoS One 9(2): e87872. doi: 10.1371/journal.pone.0087872

Mooney, A.C. et al. 2018. "Beyond social desirability bias: Investigating inconsistencies in self-reported HIV testing and treatment behaviors among HIV-positive adults in North West Province, South Africa," AIDS and Behavior 22(7): 23682379. doi: 10.1007/s10461-018-2155-9

National Department of Health [NDoH], Statistics South Africa [Stats SA], South African Medical Research Council [SAMRC], and ICF. 2019. South Africa Demographic and Health Survey 2016. Pretoria, South Africa, and Rockville, Maryland, USA: NDoH, Stats SA, SAMRC, and ICF.

National Treasury. 2018. Local government capital asset management guideline. Pretoria.

OANDA. Online trading and FX for Business [Internet]. 2019. Available: https://www.oanda.com

Patton MQ. 2015. Qualitative research \& evaluation methods: Integrating theory and practice. Thousand Oaks, CA: Sage.

PEPFAR, USAID, and Government of South Africa. 2015. "Country Operational Plan (COP) 2015: Strategic direction summary," Washington, DC: PEPFAR, USAID, and Government of South Africa.

Pettifor, A., et al. 2018. "Community mobilization to modify harmful gender norms and reduce HIV risk: results from a community cluster randomized trial in South Africa," Journal of the International AIDS Society 21(7): e25134. doi: 10.1002/jia2.25134

Pulerwitz J, Barker G. 2008. "Measuring attitudes toward gender norms among young men in Brazil," Men and Masculinities 10(3):322. doi: 10.1177/1097184X06298778

Pulerwitz, J., et al. 2010. "Addressing gender dynamics and engaging men in HIV programs: lessons learned from Horizons research," Public Health Reports 125(2): 282. doi: 10.1177/003335491012500219

Pulerwitz, J. et al. 2019 "Gender norms and HIV testing/treatment uptake: Evidence from a large population-based sample in South Africa," AIDS and Behavior 23(Suppl 2): 162-171. doi: 10.1007/s10461-019-02603-8

Sileo, K.M. et al. 2018. “What role do masculine norms play in men's HIV testing in sub-Saharan Africa?: A scoping review," AIDS and Behavior 22: 2468-2479. doi: 10.1007/s10461-018-2160-z

South African Reserve Bank. 2018. Discount rate [Internet]. Available: https://www.resbank.co.za/Pages/default.aspx

StataCorp. 2017. Stata Statistical Software: Release 15. College Station, TX: StataCorp LLC.

Stats SA. 2018. Consumer Price Index. Pretoria.

Sweat, M. et al. 2011. "Community-based intervention to increase HIV testing and case detection in people aged 16-32 years in Tanzania, Zimbabwe, and Thailand (NIMH Project Accept, HPTN 043): a randomised study," The Lancet Infectious Diseases 11(7): 525-32. doi: 10.1016/S1473-3099(11)70060-3.

Tsai, Alexander C. and S.V. Subramanian. 2012. "Proximate context of gender-unequal norms and women's HIV risk in sub-Saharan Africa," AIDS 26(3): 381-386. doi: 10.1097/QAD.0b013e32834e1ccb

UNAIDS. 2019. “South Africa Country factsheet." Geneva: UNAIDS. https://www.unaids.org/en/regionscountries/ countries/southafrica. Accessed 25 January 2021.

UNAIDS. 2000. Costing Guidelines for HIV Prevention Strategies. Geneva: UNAIDS.

Zumbo, B.D., A.M. Gadermann, and C. Zeisser. 2007. "Ordinal versions of coefficients alpha and theta for Likert rating scales," Journal of Modern Applied Statistical Methods 6(1): 4. doi: 10.22237/jmasm/1177992180 
Project SOAR

Population Council 4301 Connecticut Ave, NW,

Suite 280

Washington, D.C. 20008 USA

Tel: +1 2022379400

Fax: +1 2022378410

Ideas. Evidence. Impact.

projsoar.org 